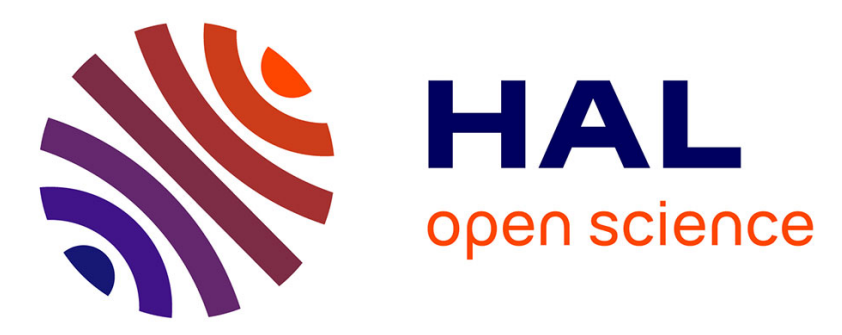

\title{
Doppler-free spectroscopy of the lowest triplet states of helium using double optical resonance
}

\author{
Ali Dia, Marie Abboud, Pierre-Jean Nacher, Geneviève Tastevin
}

\section{To cite this version:}

Ali Dia, Marie Abboud, Pierre-Jean Nacher, Geneviève Tastevin. Doppler-free spectroscopy of the lowest triplet states of helium using double optical resonance. The European Physical Journal D: Atomic, molecular, optical and plasma physics, 2021, 75, pp.223. 10.1140/epjd/s10053-021-00233-1 . hal-03295023

\section{HAL Id: hal-03295023 \\ https://hal.science/hal-03295023}

Submitted on 21 Jul 2021

HAL is a multi-disciplinary open access archive for the deposit and dissemination of scientific research documents, whether they are published or not. The documents may come from teaching and research institutions in France or abroad, or from public or private research centers.
L'archive ouverte pluridisciplinaire HAL, est destinée au dépôt et à la diffusion de documents scientifiques de niveau recherche, publiés ou non, émanant des établissements d'enseignement et de recherche français ou étrangers, des laboratoires publics ou privés.

\section{다)(1) $(5$}

Distributed under a Creative Commons Attribution - NonCommercial| 4.0 International 


\title{
Doppler-free spectroscopy of the lowest triplet states of helium using double optical resonance
}

\author{
Ali Dia ${ }^{1,2}$, Marie Abboud ${ }^{2}$, Pierre-Jean Nacher ${ }^{* 1}$, and Geneviève Tastevin ${ }^{1}$ \\ ${ }^{1}$ Laboratoire Kastler Brossel, ENS-PSL Research University, CNRS, UPMC-Sorbonne Université, Collège de France; 24 rue \\ Lhomond, F-75005 Paris, France. \\ ${ }^{2}$ Physics Department, UR TVA, Faculty of Science, Saint Joseph University, BP 11-514 Riad El Solh, Beirut 11072050, \\ Lebanon
}

\begin{abstract}
Optical pumping on the $2^{3} \mathrm{~S}-2^{3} \mathrm{P}$ transition $(1083 \mathrm{~nm})$ of metastable ${ }^{3} \mathrm{He}$ or ${ }^{4} \mathrm{He}$ atoms is used for science and applications. We report on its combination with an optical probe on the $2^{3} \mathrm{P}-3^{3} \mathrm{~S}$ transition $(706.5 \mathrm{~nm})$ in a ladder-type double optical resonance scheme, with cw single-frequency pump and probe diode lasers. Theoretical expectations for level structure and absorption spectra were computed for both isotopes. Narrow Doppler-free spectral lines were experimentally obtained in low-pressure gas samples. The line weights and their Zeeman structure in weak magnetic field agreed with expectations. The precision of the line positions and line splittings was limited by generic errors of our commercial Fizeau wavemeter, which were independently characterised. The potential of this double resonance scheme for spectroscopic measurements on the three lowest triplet states of He was evaluated. The relevance of velocity- and sublevel-selective pumping combined with polarisation spectroscopy of probe absorption for a study of pressure-dependent population transfers in the $2^{3} \mathrm{P}$ state was established.
\end{abstract}

\section{Introduction}

The two lowest-lying triplet electronic states of helium are involved in the $1083 \mathrm{~nm}$ optical transition used to perform optical pumping (OP) of the $2^{3} \mathrm{~S}$ metastable state, which is usually populated by a radiofrequency ( $\mathrm{RF}$ ) discharge in a low-pressure gas. In ${ }^{3} \mathrm{He}$, OP is the driving term of metastability-exchange optical pumping (MEOP), an efficient process yielding high nuclear polarisations in the atomic ground state and used in many applications, ranging from fundamental physics to medical imaging [1. Additional features resulting from polarisation in the $2^{3} \mathrm{~S}$ state are also investigated in gas discharges in ${ }^{3} \mathrm{He}$ [2, 3, 4] or used in ${ }^{4} \mathrm{He}$ for a variety of magnetometer schemes [5, 6, 7]. One important physical process affecting the operation and efficiency of the $2^{3} \mathrm{~S}-2^{3} \mathrm{P}$ OP cycle is the redistribution among sublevels of the $2^{3} \mathrm{P}$ state during the radiative lifetime as a result of collisions with groundstate atoms of the gas. Its pressure-dependent influence may be strong, yielding for instance a change in the sign of the OP-induced polarisation between low and high gas pressure [1, Sect. III.C.2].

In spite of the strong influence of these collisions, detailed studies are still missing and only few theoretical evaluations [8, 9] and one global indirect measurement of collision rates [10, have been reported. We therefore designed an experiment to measure atomic populations and their velocity distributions in the $2^{3} \mathrm{P}$ state using a weak optical probe on the $2^{3} \mathrm{P}-3^{3} \mathrm{~S}$ transition at $706.5 \mathrm{~nm}$ in gaseous samples submitted to velocity-selective OP on the $2^{3} \mathrm{~S}-2^{3} \mathrm{P}$ transition at $1083 \mathrm{~nm}$. A collinear and copropagating pump-probe configuration was chosen. Ab-

\footnotetext{
*nacher@lkb.ens.fr, corresponding author.
}

sorption spectroscopy was used for quantitative assessment of atomic number densities for the selectively excited atoms and for atoms having changed velocity or sublevel as well.

Initial evidence of the effect of collisions observed in such experiments was reported for isotopic mixtures [11, and the results of a detailed study will be reported elsewhere. In this article we mainly focus on low-pressure situations, in which the effect of collisions is weak, and report on Doppler-free measurements involving the $2^{3} \mathrm{P}$ $3^{3} \mathrm{~S} 706.5 \mathrm{~nm}$ transition in ${ }^{3} \mathrm{He}$ and ${ }^{4} \mathrm{He}$. We also display an example of $706.5 \mathrm{~nm}$ absorption spectrum at higher pressure for comparison with existing methods: analyses of spectra emitted by gas discharges, used to extract hyperfine structures of ${ }^{3} \mathrm{He}$ and isotope shifts [12, 13, pressure broadening [14] and pressure shift [15] in ${ }^{4} \mathrm{He}$, and optogalvanic detection of the Doppler-broadened absorption spectra of a $706.5 \mathrm{~nm}$ probe laser by a ${ }^{3} \mathrm{He}$ discharge cell [16].

The structure of this article is the following: In Sect. 2 we introduce notations and list relevant computed data, derive the computed amplitude of double optical resonance signals, and discuss their expected linewidths and Zeeman structures. Section 3 describes the experimental setup and Sect. 4 compiles experimental results obtained with pure or mixed helium isotopes at various pressures. Errors introduced by our wavemeter in spectroscopic measurements are characterised (Sect. 4.2 and Appendix D, examples of Zeeman spectra are presented, the influence of pump intensity on probe absorption is reported, and the potential of the method and setup for Doppler-free spectroscopy is evaluated. Finally, the current limits and assets of double optical resonance of metastable helium are discussed in Sect. 5 . 


\section{Theory}

\subsection{Atomic structures and optical transi- tions}

The structures of the three lowest triplet states of helium involved in the ladder-type pump-probe experiments reported in this work are schematically depicted in Fig. 1 for both helium isotopes. The fine or hyperfine structures of the $2^{3} \mathrm{~S}$ and $2^{3} \mathrm{P}$ states give rise to the energy shifts listed in Table A.1 for the sublevels of given $J$-values (for ${ }^{4} \mathrm{He}$ ) or $F$-values (for ${ }^{3} \mathrm{He}$ ) involved in the $1083 \mathrm{~nm}$ optical transition. The remaining degeneracies between magnetic sublevels are removed when a field $B$ is applied and the Zeeman effect becomes significant. The sublevel naming conventions depicted in Fig. 1, the magnetic-field-dependence of their energies, and the weights of the optical transitions between them can be found in Ref. 17]. They are recalled for the sake of convenience in Appendix $\mathrm{A}$ in the case of low magnetic field and linear Zeeman effect.

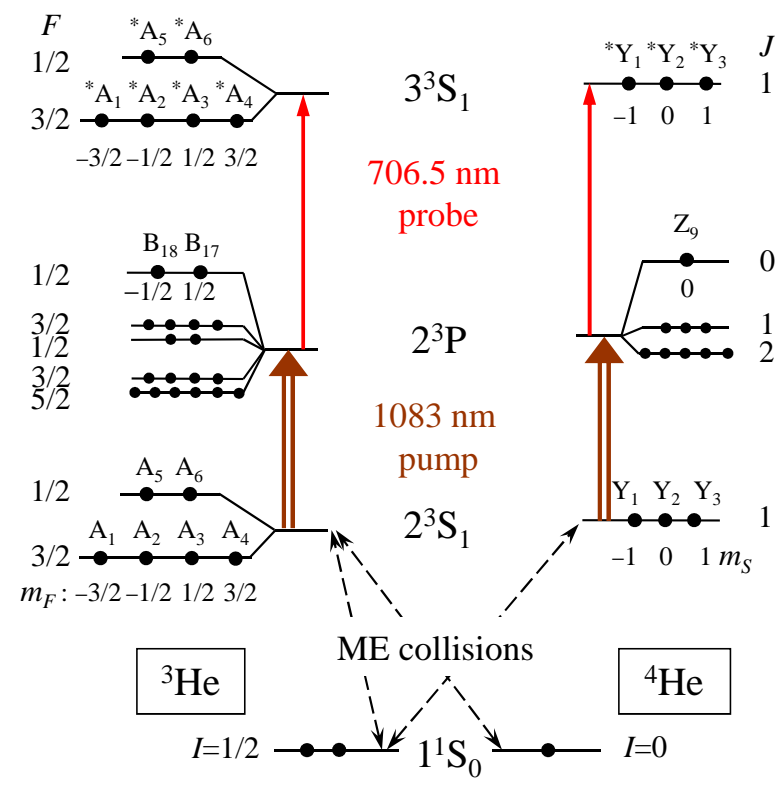

Figure 1: Ground-state and lowest triplet energy levels of ${ }^{3} \mathrm{He}$ (left) and ${ }^{4} \mathrm{He}$ (right) in null magnetic field. The energy differences between atomic states and between levels characterised by their electronic $(J)$ or total $(F)$ angular momentum are not displayed to scale. The Zeeman sublevels with angular momentum projections $m_{J}$ or $m_{F}$ are represented by bullet symbols. For clarity the sublevel names are indicated for the ${ }^{3} \mathrm{~S}$ and $2^{3} \mathrm{P}_{0}$ states only (the full lists for the $2^{3} \mathrm{P}$ state are found in Ref.[17]). The pump and probe transitions are sketched between states, but they actually address well-defined sublevels depending on the frequency and polarisation of the corresponding lasers. Metastability-exchange (ME) collisions are displayed for completeness. They play no direct role in the optical transitions but tend to enforce a spin-temperature distribution of the $2^{3} \mathrm{~S}$ state sublevel populations when ${ }^{3} \mathrm{He}$ is present in the gas.
The structure of the $3^{3} \mathrm{~S}$ state is indeed similar to that of the metastable $2^{3} \mathrm{~S}$ state and differs mostly because of the $\sim 3 \%$ lower value of its hyperfine structure constant (Eq. A.1. We therefore systematically use similar notations for all quantities related to these two states, with a star added as left upper index to denote those pertaining to atomic sublevels of the $3^{3} \mathrm{~S}$ state (such as the sublevel names in Fig. 11 or to $706.5 \mathrm{~nm}$ line components. For example, we name ${ }^{*} \mathrm{D}_{0}$ to ${ }^{*} \mathrm{D}_{2}$ the three line components connecting the $2^{3} \mathrm{P}_{0}$ to $2^{3} \mathrm{P}_{2}$ levels to the $3^{3} \mathrm{~S}$ state of ${ }^{4} \mathrm{He}$, and ${ }^{*} \mathrm{C}_{1}$ to ${ }^{*} \mathrm{C}_{9}$ the nine line components of the $706.5 \mathrm{~nm}$ transition in ${ }^{3} \mathrm{He}$.

Using the same approach as in Ref [17] the level structure of the $3^{3} \mathrm{~S}$ state is computed in the decoupled representations ( $L, S$ for ${ }^{4} \mathrm{He}$ or $L, S, I$ for ${ }^{3} \mathrm{He}$ ). Transition matrix elements ${ }^{*} T_{j k}^{(4)}\left(e_{\lambda}\right)$ for ${ }^{4} \mathrm{He}$ between sublevels $\mathrm{Z}_{j}$ and ${ }^{*} \mathrm{Y}_{k}$ and ${ }^{*} T_{j k}\left(e_{\lambda}\right)$ for ${ }^{3} \mathrm{He}$ between sublevels $\mathrm{B}_{j}$ and ${ }^{*} \mathrm{~A}_{k}$ are computed for the three light polarisation states $e_{\lambda}$ $\left(\sigma_{+}, \sigma_{-}\right.$, and $\left.\pi\right)$. For both optical transitions the matrix elements correspond to branching ratios for radiative decay. Partial sums over the lower sublevels for each upper sublevel in the $3^{3} \mathrm{~S}$ state therefore verify

$$
\sum_{j, e_{\lambda}}{ }^{*} T_{j k}^{(4)}\left(e_{\lambda}\right)=\sum_{j, e_{\lambda}}{ }^{*} T_{j k}\left(e_{\lambda}\right)=1 .
$$

Additionally, the ${ }^{4} \mathrm{He}$ matrix elements of the two transitions verify exact relations:

$$
{ }^{*} T_{j k}^{(4)}\left(\sigma_{ \pm}\right)=T_{k j}^{(4)}\left(\sigma_{\mp}\right) / 3,{ }^{*} T_{j k}^{(4)}(\pi)=T_{k j}^{(4)}(\pi) / 3,
$$

where the opposite helicities of light result from the opposite roles (upper or lower level) of the $3^{3} \mathrm{~S}$ and $2^{3} \mathrm{~S}$ states in the optical transitions. The ${ }^{3} \mathrm{He}$ matrix elements verify similar relations only for $B=0$, with small differences (for instance of order $10^{-3}$ at $B=0.1 \mathrm{~T}$ ) resulting from the difference in hyperfine structure constant values (Eq. A.1)

$$
{ }^{*} T_{j k}\left(\sigma_{ \pm}\right) \approx T_{k j}\left(\sigma_{\mp}\right) / 3,{ }^{*} T_{j k}(\pi) \approx T_{k j}(\pi) / 3 .
$$

For the $1083 \mathrm{~nm}$ transition the energies of the line components are referred to that of $\mathrm{C}_{1}(B=0)$ and the differences are generically noted $\varepsilon$ or $\varepsilon^{(4)}$, with one index (that of the line component) or two indices (those of the relevant sublevels). We use a similar convention for the $706.5 \mathrm{~nm}$ transition, with energy differences ${ }^{*} \epsilon$ and ${ }^{*} \epsilon{ }^{(4)}$ to that of ${ }^{*} \mathrm{C}_{1}(B=0)$, in this case the highest-lying energy. Computed values of ${ }^{*} \epsilon / h$, of ${ }^{*} \epsilon^{(4)} / h$, of ${ }^{*} T_{j k}$, and of ${ }^{*} T_{j k}^{(4)}$ at null field are tabulated in Tabs. A.2 to A.5 and used in Figs. 2a and 2 p.

Another significant difference between the $2^{3} \mathrm{~S}$ and $3^{3} \mathrm{~S}$ levels is the value of the isotope shift (Eq. A.6 or A.7 for the $3^{3} \mathrm{~S}$ state) which is used to compute transition energy differences $\varepsilon^{(4)}$ and ${ }^{*} \epsilon^{(4)}$. It results in fully overlapping Doppler-broadened spectra (Fig. 2p and 12, 13]) for the $706.5 \mathrm{~nm}$ lines, a feature which contrasts with the large isotope shift between $1083 \mathrm{~nm}$ lines (Fig. 2d).

Finally, the Landé $g$-factors for the two states (Table A.1 only very slightly differ (by about 1 ppm, [18, Table 5]), which results in very similar Zeeman effects. 

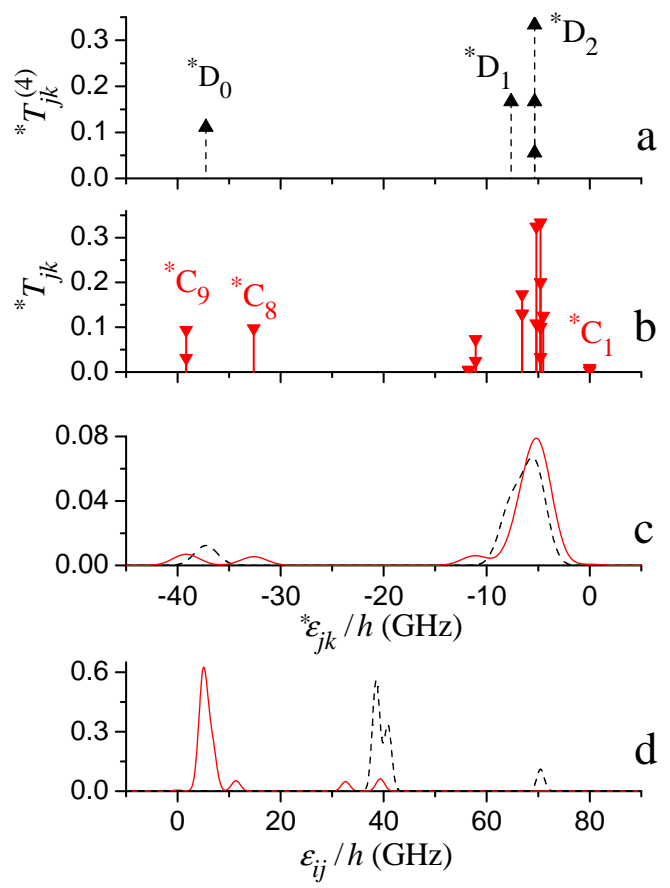

Figure 2: Computed transition matrix elements and spectral functions for the $706.5 \mathrm{~nm}$ (a to c) and $1083 \mathrm{~nm}(\mathrm{~d})$ transitions at $B=0$. a: ${ }^{*} T_{j k}^{(4)}$ and b: ${ }^{*} T_{j k}$ values for circular polarisation are plotted vs. the shifts from ${ }^{*} \mathrm{C}_{1}$ (for ${ }^{4} \mathrm{He}$ and ${ }^{3} \mathrm{He}$, respectively). The names of corresponding lines of special interest are indicated. Several symbols at the same shift correspond to transitions between distinct degenerate Zeeman sublevels (Tables A.3 and A.5. c: Corresponding scaled spectral functions ${ }^{*} \Delta_{3} \sqrt{\pi}^{*} S_{\text {th }}$ and ${ }^{*} \Delta_{4} \sqrt{\pi}{ }^{*} S_{\text {th }}^{(4)}$ (see Eq. 13 for thermal velocity distributions $(T=300 \mathrm{~K})$. d: Scaled spectral functions $\Delta_{3} \sqrt{\pi} S_{\mathrm{th}}$ and $\Delta_{4} \sqrt{\pi} S_{\text {th }}^{(4)}$ for the $1083 \mathrm{~nm}$ transition, displaying mirrored features with a much larger isotope shift (note the twice larger horizontal scale) and better-resolved line components due to the smaller Doppler widths at larger wavelength.

\subsection{Probe transmittance}

Explicit expressions for the transmittance $\mathcal{T}$ of a weak probe beam through a helium gas sample can be written when several simplifying assumptions are made: The probe light has one of the three polarisation states $e_{\lambda}$, the probe beam has a small transverse size, across which the populations of all states are uniform, and the probe intensity is weak enough for stimulated emission to be negligible. In this article the probe beam is tuned to the $706.5 \mathrm{~nm}$ transition but most results in this section apply for a weak probe on the $1083 \mathrm{~nm}$ transition as well. We therefore use generic notations for the lower (L) and higher $(\mathrm{H})$ states of a transition in an isotope, with degeneracies $g_{\mathrm{L}}$ and $g_{\mathrm{H}}$, sublevels $\mathrm{L}_{p}$ and $\mathrm{H}_{q}$, and populations $l_{p}$ in the lower state. Note that $l_{p}$ are truly populations (with $\sum a_{i}=\sum y_{i}=1$ ) only for the $2^{3} \mathrm{~S}$ state. The (pseudo)populations $b_{j}, z_{j},{ }^{*} a_{k}$, and ${ }^{*} y_{k}$ are conve- nient parameters to obtain the number densities of the corresponding $2^{3} \mathrm{P}$ state sublevels from $\hat{n}$, as detailed in Table A.4. The local metastable number density $\hat{n}$ stands for the isotope-specific notations $n_{\mathrm{m}}$ or $n_{\mathrm{m}}^{(4)}$ 17. We also introduce hat-decorated symbols for all quantities and names that may consistently pertain to the ${ }^{3} \mathrm{He}$ or ${ }^{4} \mathrm{He}$ isotope, as well as to to the 706.5 or $1083 \mathrm{~nm}$ transition, in text or equations. $\hat{T}_{p q}$ are the transition matrix elements that represent $T_{i j}$ or $T_{i j}^{(4)}$ for pump light absorption and ${ }^{*} T_{p q}$ or ${ }^{*} T_{p q}^{(4)}$ for probe light absorption, for instance.

The probe light intensity $I_{\mathrm{pr}}$. decreases with $z$ when propagating in the sample because of absorbed and scattered light

$$
-\frac{d I_{\mathrm{pr} .}}{d z}=h \nu_{\mathrm{pr} .} \hat{n} \sum_{p, q} l_{p} \int d v_{z} \phi_{\mathrm{L} p}\left(v_{z}\right) \hat{\gamma}_{p q}\left(v_{z}\right),
$$

in which $\nu_{\mathrm{pr}}$. is the central probe laser frequency, $\phi_{\mathrm{L} p}$ is the normalised velocity distribution in sublevel $\mathrm{L}_{p}$, and $\hat{\gamma}_{p q}$ is the velocity-dependent optical transition rate from $\mathrm{L}_{p}$ to $\mathrm{H}_{q}$. For a normalised frequency distribution $\mathcal{F}\left(\nu-\nu_{\text {pr. }}\right)$ of the probe light intensity, the transition rates may be written as

$$
\begin{aligned}
\hat{\gamma}_{p q}\left(v_{z}\right) & =\hat{K} I_{\text {pr. }} \hat{T}_{p q} \\
& \times \int d \nu \mathcal{F}\left(\nu-\nu_{\text {pr. }}\right) \mathcal{L}_{W}\left[\nu-\frac{\hat{\epsilon}_{p q}}{h}\left(1-\frac{v_{z}}{c}\right)\right]
\end{aligned}
$$

in which $\mathcal{L}_{W}$ is the normalised Lorentzian distribution of width $W$ :

$$
\mathcal{L}_{W}\left(\nu-\nu^{\prime}\right)=\frac{2 /(\pi W)}{1+\left[2\left(\nu-\nu^{\prime}\right) / W\right]^{2}} .
$$

In Eq. 5 the FWHM (in frequency units) $W=\hat{\Gamma}^{\prime} / 2 \pi$ is linked with the pressure-broadened damping rate of the $\mathrm{L}-\mathrm{H}$ transition. The coefficient $\hat{K}$ can be conveniently derived by noticing that each integral over velocities in the right-hand side sum of Eq. 4 is a convolution of normalised functions, and is thus a normalised function of the probe frequency. For an unpolarised gas with equally populated sublevels $\left(\hat{n} l_{p}=\hat{n}_{\mathrm{L}} / g_{\mathrm{L}}\right.$, with $\hat{n}_{\mathrm{L}}$ the number density in the lower level), Eq. 4 can therefore be identified with the usual expression of absorption cross section [19]

$$
\sigma_{a}\left(\nu_{\text {pr. }}\right)=-\frac{1}{\hat{n}_{\mathrm{L}} I_{\mathrm{pr} .}} \frac{d I_{\mathrm{pr} .}}{d z}\left(\nu_{\mathrm{pr} .}\right)=\hat{\gamma} \frac{\hat{\lambda}^{2}}{8 \pi} \frac{g_{\mathrm{H}}}{g_{\mathrm{L}}} g\left(\nu_{\mathrm{pr} .}\right)
$$

where $\hat{\gamma}$ is the total rate of spontaneous emission from the higher level, $\hat{\lambda}$ the transition wavelength, and $g$ a normalised lineshape function. Summing relations like Eq. 1 over all higher sublevels but retaining only one of the three

\footnotetext{
${ }^{1}$ The difference between Eq. 7 and the expressions used in Ref. 19 results from the fact that optical angular frequency $\omega$ is consistently used in that work, contrary to e.g., Ref 20 and this article in which the optical frequency $\nu$ is used. Ref. 19] was revised and some corrections were made in 21] but Eq. 7 remained unchanged.
} 
light polarisations yields $\sum_{p, q} \hat{T}_{p q}\left(e_{\lambda}\right)=g_{\mathrm{H}} / 3$, and the value of $\hat{K}$ is derived

$$
h \nu_{\text {pr. }} \hat{K}=\frac{3 \hat{\lambda}^{2} \hat{\gamma}}{8 \pi} .
$$

This can equivalently be expressed in terms of the absorption oscillator strength $\hat{f}$ as well [19]:

$$
h \nu_{\mathrm{pr} .} \hat{K}=\frac{3 g_{\mathrm{L}}}{g_{\mathrm{H}}} \frac{h \alpha \hat{f}}{2 m_{\mathrm{e}}},
$$

where $\alpha$ is the fine-structure constant and $m_{\mathrm{e}}$ the electron mass. The ratio $3 g_{\mathrm{L}} / g_{\mathrm{H}}=1$ for the $1083 \mathrm{~nm}$ transition and 9 for the $706.5 \mathrm{~nm}$ transition.

These relations can be further simplified in several situations. For instance, when the sample is optically thin or velocity distributions are sufficiently broad, absorption only affects the probe intensity $I_{\text {pr. }}(z)$, not its frequency distribution $\mathcal{F}$. In particular, if $\mathcal{F}=\mathcal{L}_{\delta}$ is a Lorentzian intensity distribution of width $\delta$, the convolution in Eq. 5 simply yields a Lorentzian of width $W+\delta$ :

$$
\hat{\gamma}_{p q}\left(v_{z}\right)=\hat{K} I_{\text {pr. }}(z) \hat{T}_{p q} \mathcal{L}_{W+\delta}\left[\nu_{\text {pr. }}-\frac{\hat{\epsilon}_{p q}}{h}\left(1-\frac{v_{z}}{c}\right)\right] .
$$

The probe transmittance is finally derived by integration on $z$ over the cell length, $L_{\text {cell }}$, of the right-hand side of Eq. 4 divided by $I_{\text {pr. }}$. This simply introduces spatial averages $\left\langle\hat{n} l_{p}\right\rangle$ of the probed sublevel atomic densities (or the spatial average $\langle\hat{n}\rangle$ if the populations are uniform, which is assumed in the following), and a polarisation-dependent spectral function noted $\hat{S}$ :

$$
\begin{aligned}
-\ln \hat{\mathcal{T}} & =L_{\text {cell }}\langle\hat{n}\rangle \frac{3 \hat{\lambda}^{2} \hat{\gamma}}{8 \pi} \hat{S}\left(\nu_{\text {pr. }}\right), \text { with } \\
\hat{S}(\nu) & =\sum_{p, q} l_{p} \hat{T}_{p q} \int d v_{z} \phi_{\mathrm{L} p}\left(v_{z}\right) \mathcal{L}_{W+\delta}\left[\nu-\frac{\hat{\epsilon}_{p q}}{h}\left(1-\frac{v_{z}}{c}\right)\right]
\end{aligned}
$$

For thermal velocity distributions in unpolarised gas, which occur in a weak discharge in the absence of pump beam, the thermal spectral functions $\hat{S}_{\text {th }}$ are linear combinations of Voigt profiles. For low gas pressure and narrowband probe light $(W+\delta \ll \hat{\Delta}$, the Doppler width defined in Appendix B, with values listed in Table B.1), $\hat{S}_{\text {th }}$ approximately combines Gaussian profiles:

$$
\hat{S}_{\mathrm{th}}(\nu) \approx \frac{1}{\hat{\Delta} \sqrt{\pi}} \sum_{p, q} l_{p} \hat{T}_{p q} \exp -\frac{\left(\nu-\hat{\epsilon}_{p q} / h\right)^{2}}{\hat{\Delta}^{2}} .
$$

The scaled dimensionless quantities $\hat{\Delta} \sqrt{\pi} \hat{S}_{\text {th }}$ are plotted in Figs. 2e and 2d. For the $1083 \mathrm{~nm}$ transition this quantitative link between transmittance and number densities of absorbers in the relevant sublevels is indeed identical to that previously published [17, 20, 22]. It applies to the $706.5 \mathrm{~nm}$ transition as well when replacing the generic parameters in Eqs. 11 and 13 with the specific ones.
When velocity distributions $\phi_{\mathrm{L} p}\left(v_{z}\right)$ depart from thermal equilibrium, as discussed in the next section, $\hat{S}$ retains the property that each integral over $v_{z}$ is a normalised function of frequency which means for instance that integration of resolved components of transmittance data can yield quantitative information of the corresponding sublevel number densities. For instance, when the ${ }^{*} \mathrm{D}_{0}$ line component is scanned in ${ }^{4} \mathrm{He}$ using any probe polarisation, the area ${ }^{*} \mathcal{A}_{0}^{(4)}$ of the absolute transmittance data is

$$
\begin{aligned}
{ }^{*} \mathcal{A}_{0}^{(4)} & =-\int_{{ }^{*} \mathrm{D}_{0}} d \nu_{\text {pr. }} \ln { }^{*} \mathcal{T}\left(\nu_{\text {pr. }}\right) \\
& =L_{\text {cell }}\left\langle n_{\mathrm{m}}^{(4)}\right\rangle \frac{3^{*} \lambda^{2 *} \gamma}{8 \pi} T_{9 k}^{(4)} z_{9}
\end{aligned}
$$

where the index $\mathrm{k}$ depends on the choice of polarisation but all ${ }^{*} T_{9 k}^{(4)}$ are equal to $1 / 9$ (the values of transition matrix elements ${ }^{*} T_{j k}^{(4)}$ and ${ }^{*} T_{j k}$ are listed in Tables A.3 and A.5. For other lines, similar simple relations can be explicitly derived in particular for a $\pi$-polarised probe, a configuration experimentally studied in this work. When the ${ }^{*} \mathrm{D}_{1}$ component is used, the populations $z_{6}$ and $z_{8}$ are probed with equal weights and

$$
{ }^{*} \mathcal{A}_{1}^{(4)}(\pi)=L_{\text {cell }}\left\langle n_{\mathrm{m}}^{(4)}\right\rangle \frac{3^{*} \lambda^{2 *} \gamma}{8 \pi} T_{61}^{(4)}\left(z_{6}+z_{8}\right) .
$$

Similarly when the ${ }^{*} \mathrm{C}_{n}$ line components probe absorption by ${ }^{3} \mathrm{He}$ atoms in the $2^{3} \mathrm{P}_{0}$ level $(n=8$ or 9$)$

$$
{ }^{*} \mathcal{A}_{n}(\pi)=L_{\text {cell }}\left\langle n_{\mathrm{m}}\right\rangle \frac{3{ }^{*} \lambda^{2 *} \gamma}{8 \pi} T_{18, k}\left(b_{18}+b_{17}\right),
$$

where $k=5$ for $n=8$ and $k=2$ for $n=9$.

These expressions relate probe absorption data to number densities of selected sublevels in the $2^{3} \mathrm{P}$ state. Besides, the total number density in the $2^{3} \mathrm{P}$ state can independently be linked with the absorbed pump intensity $\Delta I_{\mathrm{P}}$ using simple detailed balance considerations involving the $2^{3} \mathrm{P}$ decay rate and the wavelength of the pumping transition:

$$
\Delta I_{\mathrm{P}}=L_{\text {cell }}\left[\left\langle n_{\mathrm{m}}\right\rangle \sum_{j=1}^{18} b_{j}+\left\langle n_{\mathrm{m}}^{(4)}\right\rangle \sum_{j=1}^{9} z_{j}\right] \gamma h c / \lambda .
$$

Discharge-dependent parameters are thus eliminated when considering the ratios of any of the line areas $* \hat{\mathcal{A}}$ (Eqs. 14 to 16 to the absorbed pump intensity (Eq. 17), which yields

$$
\begin{aligned}
\left({ }^{*} \hat{\mathcal{A}} /{ }^{*} \hat{T}_{j k}\right) / \Delta I_{\mathrm{P}} & =\frac{3^{*} \lambda^{2 *} \gamma \lambda}{8 \pi \gamma h c} \hat{\mathcal{R}} \\
\text { with } \frac{3^{*} \lambda^{2 *} \gamma \lambda}{8 \pi \gamma h c} & =8.843 \mathrm{MHz} \mathrm{cm}^{2} \mathrm{~mW}^{-1},
\end{aligned}
$$

where $\hat{\mathcal{R}}$ is the fraction of the atoms in the $2^{3} \mathrm{P}$ state which are in the probed sublevel(s), e.g., $\mathcal{R}_{0}=z_{9} / \sum_{j=1}^{9} z_{j}$ for a probe scanning the ${ }^{*} \mathrm{D}_{0}$ line in pure ${ }^{4} \mathrm{He}$. 
When the pump intensity is weak enough to induce negligible atomic alignment or orientation (population differences) in the $2^{3} \mathrm{~S}$ state and small enough populations in the $2^{3} \mathrm{P}$ state (therefore negligible stimulated emission), an exponential intensity decrease results from absorption in the gas. In that limit, the absorbed pump intensity linearly scales with the average pump intensity as

$$
\Delta I_{\mathrm{P}}^{(\operatorname{lin})}=-\ln \mathcal{T}_{0}<I_{\mathrm{P}}>
$$

where $\mathcal{T}_{0}$ is the null-intensity limit transmittance of the pump beam. When Eq. 20 is substituted in Eq. 18, a linear increase of the areas ${ }^{*} \hat{\mathcal{A}}$ with the average pump intensity is obtained in the weak-pumping limit. It involves the factor in Eq. 19 and the fraction $\hat{\mathcal{R}}$ which is of the order of 1 at low enough pressure:

$$
{ }^{*} \hat{\mathcal{A}}^{(\operatorname{lin})}=-\ln \mathcal{T}_{0}{ }^{*} \hat{T}_{j k} \hat{\mathcal{R}} \frac{3^{*} \lambda^{2} \gamma \lambda}{8 \pi \gamma h c}<I_{\mathrm{P}}>.
$$

This sets the scale for the expected signals and is used for comparisons with experimental data.

\subsection{VSOP and probe absorption linewidth}

Velocity-selective optical pumping (VSOP, 23) is the key first step of double optical resonance. With a narrow pump laser tuned close to an atomic resonance, only atoms with velocity projections close to zero are excited with a significant rate from the $2^{3} \mathrm{~S}$ to the $2^{3} \mathrm{P}$ state. Velocity distribution profiles in these two states may therefore strongly differ from thermal distributions. This is illustrated in Fig. 3 for the simple case of isolated pump and probe transitions in ${ }^{4} \mathrm{He}$, namely with $\pi$-polarised pump light on the $\mathrm{D}_{0}$ component (Fig. 3 a).

The steady-state result of VSOP in helium can be described by the ( $z$-position-dependent) populations and the associated normalised velocity distributions of the $2^{3} \mathrm{~S}$ and $2^{3} \mathrm{P}$ sublevels, using the notations listed in Table A.4. For the sublevels $\mathrm{Y}_{2}$ and $\mathrm{Z}_{9}$ directly addressed by the pumping light in Fig. 3, typical velocity distributions are displayed in panels b and c, respectively. A narrow hole is burnt in the thermal velocity profile of the $\mathrm{Y}_{2}$ level (a narrow peak correspondingly appears on top of thermal velocity profiles of levels $Y_{1}$ and $Y_{3}$, not shown), while populations are modified $\left(a_{2}<a_{1}, a_{3}\right)$. On the contrary, the velocity distribution in the $2^{3} \mathrm{P}$ state mostly results from the VSOP process, with a negligible thermal contribution from the radiative cascade in a weak discharge: it is characterised by a narrow peak with a negligible broad pedestal ${ }^{2}$ These usual features of VSOP are directly relevant for the Doppler-free spectroscopy performed in this work, since the linewidth $\Delta^{*} \nu$ of scanned probe absorption signals (Fig. 3d) results from the velocity distribution width $\Delta v_{z}$ of the probed level.

\footnotetext{
${ }^{2}$ In our experimental conditions, probe absorption in the absence of pump light was too weak to be detected. Additionally, the pump beam was mechanically modulated and pump-induced probe absorption difference was extracted using lock-in detection.
}
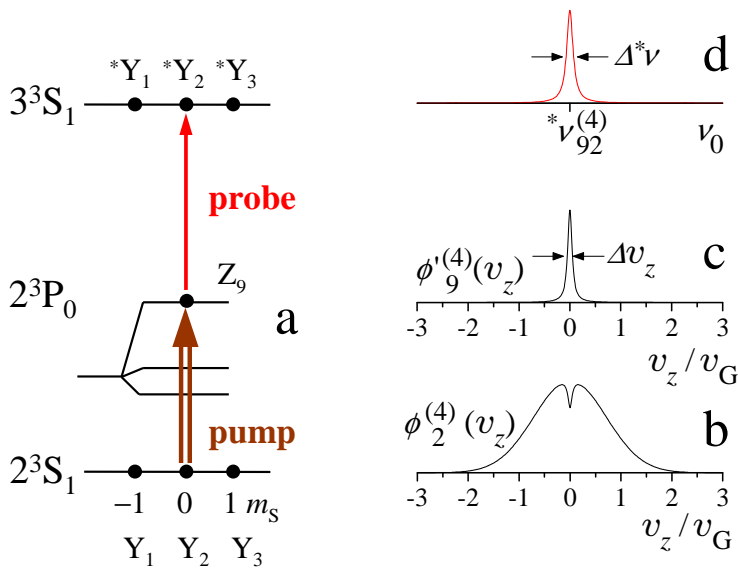

Figure 3: a: Example of double optical resonance scheme in ${ }^{4} \mathrm{He}$ using $\pi$-polarised pump and probe tuned to the $\mathrm{D}_{0}$ and ${ }^{*} \mathrm{D}_{0}$ transitions, respectively. b, c: VSOP-controlled velocity distribution profiles in the $\mathrm{Y}_{2}$ and $\mathrm{Z}_{9}$ sublevels addressed by the pump (notations are defined in Table A.4). d: Absorption spectrum for the $706.5 \mathrm{~nm}$ probe scanning the ${ }^{*} \mathrm{D}_{0}$ line. The velocity and frequency widths are discussed in the text.

If the pump intensity $I_{\mathrm{P}}$ is low enough, $\Delta v_{z}$ solely results from the combination of the natural linewidth of the $1083 \mathrm{~nm}$ transition, of its pressure broadening, and of the pump laser spectral width $\delta$. If the latter is assumed to have a Lorentzian profile, all contributions simply add up to yield the FWHM of the velocity profile for $I_{\mathrm{P}}=0$

$$
\left.\Delta v_{z}\right|_{I_{\mathrm{P}}=0}=\lambda[\gamma+2 w+2 \pi \delta] / 2 \pi
$$

The wavelength $\lambda$ of the pump in Eq. 22 converts frequencies to velocities, $\gamma$ is the radiative decay rate in the $2^{3} \mathrm{P}$ state $(\gamma / 2 \pi=1.626 \mathrm{MHz}$, 24, Table 14]) and $w$ is the pressure-dependent collision broadening rate $(w / \pi \approx 12$ to $18 \mathrm{MHz} / \mathrm{mbar},[9,22])$. The line shape of the $706.5 \mathrm{~nm}$ transition is a narrow Lorentzian profile which results (by convolution) from the velocity distribution profile in the pumped $2^{3} \mathrm{P}$ level and the linewidth of the probed transition itself. Its total width is therefore the sum of two terms, here expressed in frequency units:

$$
\left.\Delta^{*} \nu\right|_{I_{\mathrm{P}}=0}=\left.\Delta v_{z}\right|_{I_{\mathrm{P}}=0} /{ }^{*} \lambda+\Gamma_{706}^{\prime} / 2 \pi \text {. }
$$

The first part involves the width of the velocity distribution of atoms excited by the pump light obtained in Eq. 22 . converted to frequency using the wavelength ${ }^{*} \lambda$ of the probe. The second part, $\Gamma_{706}^{\prime} / 2 \pi$, combines widths arising from the radiative lifetimes of the $2^{3} \mathrm{P}$ and $3^{3} \mathrm{~S}$ states (1.626 MHz and 4.425 MHz [24, Table 14]), collisional broadening of the $706 \mathrm{~nm}$ transition (33 MHz/mbar, 14]), and the instrumental linewidth of the probe laser. Altogether, the expected width is the sum of contributions due to the radiative lifetimes $(6.92 \mathrm{MHz})$, collisional broadening (52 to $61 \mathrm{MHz} / \mathrm{mbar}$ ), and laser widths (typ. $7.5 \mathrm{MHz}$ for pump and probe lasers having $3-\mathrm{MHz}$ widths), adding 
up to $\left.\Delta^{*} \nu\right|_{I_{\mathrm{P}}=0}=22 \mathrm{MHz}$ for $0.13 \mathrm{mbar}$, the lowest pressure used in our experiments.

At sufficient pump intensity, power broadening arising from saturation of the transition and from the creation of holes in velocity profiles is well documented for simple two-level systems [25]. Dedicated OP models are needed in the case of helium to establish a quantitative link between pump intensity and broadening of the velocity profiles in the $2^{3} \mathrm{~S}$ and $2^{3} \mathrm{P}$ states. Such models usually involve a coarse phenomenological treatment of collisions and of relaxation [17, 26, 27] and their use to evaluate velocity profiles falls beyond the scope of this article. Let us only mention that pressure broadening of the velocity distribution is expected to retain its generic expression as a function of the pump intensity $I_{\mathrm{P}}$

$$
\Delta v_{z}=\left.\sqrt{1+I_{\mathrm{P}} / I_{\mathrm{sat}}} \Delta v_{z}\right|_{I_{\mathrm{P}}=0}
$$

where, however, $I_{\text {sat }}$ is an effective saturation intensity depending on various parameters in the OP model.

\subsection{Zeeman effects in double optical res- onance}

In this section generic notations are used to correspond to ${ }^{3} \mathrm{He}$ or ${ }^{4} \mathrm{He}$, but the higher and lower states are implied by the choice of the pump or probe transition. Considering a pump-induced transition from $\mathrm{L}_{i}$ to $\mathrm{H}_{j}$, with average pump frequency $\nu_{\mathrm{P}}$ and level energy difference $h \hat{\nu}_{i j}$, the pump detuning $\hat{\nu}_{i j}-\nu_{\mathrm{P}}$ selects atoms in the $2^{3} \mathrm{~S}$ state which are suitably Doppler-shifted. Their average velocity $v_{z}^{\mathrm{S}}$ and the corresponding velocity of the atoms in the $2^{3} \mathrm{P}$ state are

$$
v_{z}^{\mathrm{P}}-v_{\text {recoil }}=v_{z}^{\mathrm{S}}=\left(\hat{\nu}_{i j}-\nu_{\mathrm{P}}\right) \lambda
$$

where the recoil velocity $v_{\text {recoil }}=h /(\hat{M} \lambda)$ amounts to 12.3 and $9.2 \mathrm{~cm} / \mathrm{s}$ for ${ }^{3} \mathrm{He}$ and ${ }^{4} \mathrm{He}$, respectively $(\hat{M}$ is the atomic mass). For a $2^{3} \mathrm{P}-3^{3} \mathrm{~S}$ transition frequency ${ }^{*} \hat{\nu}_{j k}$ from $\mathrm{L}_{j}$ to $\mathrm{H}_{k}$ the probe frequency $\nu_{0}$ needed for resonant absorption by atoms with the pump-selected velocity of Eq. 25 is

$$
{ }^{*} \hat{\nu}_{j k}-\nu_{0}=v_{z}^{\mathrm{P}} /{ }^{*} \lambda=\left(\hat{\nu}_{i j}-\nu_{\mathrm{P}}\right) \lambda /{ }^{*} \lambda+h /\left(\hat{M}^{*} \lambda \lambda\right)
$$

The Zeeman shift of the resonant probe absorption frequency $\nu_{0}$ in an applied field $B$ is obtained as the difference of values obtained using Eq. 26 for $B$ and a null field. In that operation, the actual value of the pump frequency and the recoil term both cancel out, and the shift is

$\nu_{0}(B)-\nu_{0}(0)={ }^{*} \hat{\nu}_{j k}(B)-{ }^{*} \hat{\nu}_{j k}(0)-\lambda /{ }^{*} \lambda\left[\hat{\nu}_{i j}(B)-\hat{\nu}_{i j}(0)\right]$

The frequency differences on the right-hand side of Eq. 27 are conveniently expressed writing the linear $B$ dependency of the energies as a function of the $\hat{m}$ quantum numbers in the three involved sublevels, characterised by the three indices $i, j$, and $k$, and the linear Zeeman shift factor is

$$
\begin{aligned}
\frac{\nu_{0}(B)-\nu_{0}(0)}{B} & =\left[\frac{\lambda}{* \lambda} \hat{g}_{1}(i) \hat{m}_{1}(i)-\frac{\lambda+{ }^{*} \lambda}{{ }^{*} \lambda} \hat{g}_{2}(j) \hat{m}_{2}(j)\right. \\
& \left.+\hat{g}_{3}(k) \hat{m}_{3}(k)\right] \mu_{\mathrm{B}}
\end{aligned}
$$

where $\hat{g}_{1}, \hat{g}_{2}$, and $\hat{g}_{3}$ are the $g$ - factors $\left(g_{F}\right.$ for ${ }^{3} \mathrm{He}, g_{S}$ or $g_{J}$ for ${ }^{4} \mathrm{He}$ ) of the involved sublevels of the $2^{3} \mathrm{~S}, 2^{3} \mathrm{P}$, and $3^{3} \mathrm{~S}$ states, respectively, and $\hat{m}_{1}, \hat{m}_{2}$, and $\hat{m}_{3}$ their angular momentum projections. Note that the signs in the weights affecting the contributions of the energy shifts in the three states in Eq. 28 are those obtained for the co-propagating beam configuration; different weights would be derived for a counter-propagating scheme, not used in this work.

Equation 28 can be explicitly written for any pump and probe line component, using the values of $g$ - factors listed in Table A.1 and immediately after Eq. A.4. For the simple scheme involving the $2^{3} \mathrm{P}_{0}$ state of ${ }^{4} \mathrm{He}$ depicted in Fig. 3 it reduces to

$$
\frac{\nu_{0}(B)-\nu_{0}(0)}{B}=\left[\frac{\lambda}{{ }^{*} \lambda} m_{S}+{ }^{*} m_{S}\right] g_{S} \mu_{\mathrm{B}}
$$

For a $\pi$-polarised pump $\left(m_{S}=0\right.$ is selected $)$, a triplet of absorption lines can potentially be observed (depending on the probe polarisation) with the usual ${ }^{*} g_{S} \mu_{\mathrm{B}}$ splitting factor $(28 \mathrm{MHz} / \mathrm{mT})$. Conversely, for a fixed $\pi$ polarisation of the probe $\left({ }^{*} m_{S}=0\right)$, three velocity classes can potentially be excited (depending on the pump polarisation) and a triplet of absorption lines can still be observed, with an enhanced splitting factor, $g_{S} \mu_{\mathrm{B}} \lambda /{ }^{*} \lambda$ $(42.9 \mathrm{MHz} / \mathrm{mT})$. When all possible pump and probe polarisations are considered, nine lines may be observed with unevenly spaced shift factors spanning $\pm 70.9 \mathrm{MHz} / \mathrm{mT}$.

\section{Experimental setup and proto- col}

The main elements of the double optical resonance experiments lying in the same vertical plane are displayed in Fig. 4. the pump and probe lasers, the co-propagating beams overlapping in the experimental cell $\mathrm{C}_{\exp }$, the light polarisation and intensity control elements, and the saturated absorption setup which uses part of the pump beam and the additional cell $\mathrm{C}_{\text {lock. }}$. They are successively described in this section, along with off-plane light beams and elements. The setup had a $1.8 \times 0.5 \mathrm{~m}^{2}$ footprint. A set of six square coils was used to control the axial magnetic field component $B_{z}$, two pairs of rectangular coils were used to control the field components transverse to the light beams, $B_{y}$ (vertical) and $B_{x}$ (horizontal, perpendicular to the plane of Fig. 4). Details on these coil sets are given in Appendix C.

Cells - Experiments were performed at room temperature with helium gas in sealed cylindrical Pyrex glass cells. Their dimensions ranged from 3 to $12 \mathrm{~cm}$ in length, 1.5 to $5 \mathrm{~cm}$ in diameter. The filling pressures ranged from 0.13 to 


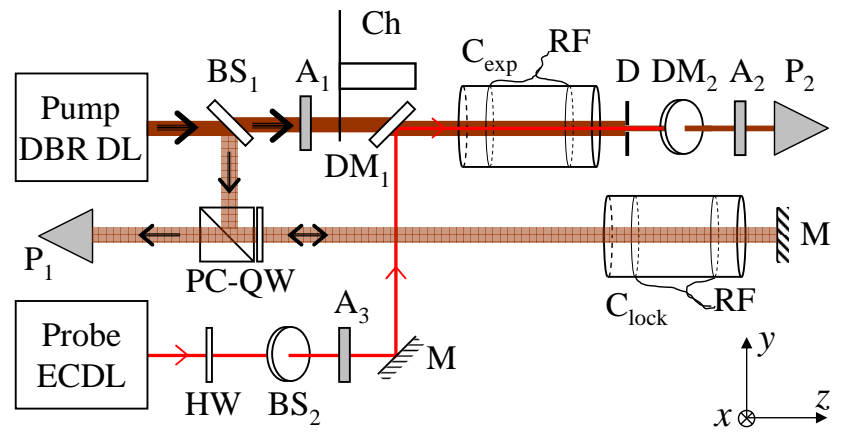

Figure 4: Schematic view of the main optical elements used in double optical resonance experiments (in the vertical $y z$ plane, not to scale). The labeled components are $10 \%$ beam samplers (BS), absorptive attenuators $(\mathrm{A})$, long-pass dichroic mirrors (DM), a polarising cube with adhered quarter-wave plate (PC-QW), a half-wave plate (HW), and mirrors (M). The optical chopper (Ch) and the iris diaphragm (D, $2.3 \mathrm{~mm}^{2}$ area) control beam propagation and diameter. Photodiode detectors $(\mathrm{P})$ monitor beam powers. $\mathrm{C}_{\text {exp }}$ is the main experimental gas cell, $\mathrm{C}_{\text {lock }}$ the ancillary cell used to lock the pump frequency on an atomic transition. Additional elements lying outside the $y z$ plane of the figure (probe wavelength measurement, transmitted probe polarisation analysis and detection, and power measurement) as well as the coil systems for $B$-field control are not displayed but are described in the text.

67 mbar of ${ }^{3} \mathrm{He},{ }^{4} \mathrm{He}$, or isotopic mixtures. The cells had been made in-house for previous experiments according to a standard cleaning and filling protocol [17, 22]. They were positioned with a small tilt angle with respect to the light beams to avoid large overlap from the reflections on the uncoated cell windows. A weak RF discharge was used to populate the $2^{3} \mathrm{~S}$ state with external wire electrodes arranged according to the cell shape. The RF frequency was of order $2-3 \mathrm{MHz}$ or $20-25 \mathrm{MHz}$, depending on cell size and gas pressure. Low RF frequencies usually provided stable operation for weak discharges and lower RF interference noise whereas higher frequencies were used for easier discharge ignition at the lowest gas pressures. The experimental cell was located at the centre of the coil sets and $\mathrm{C}_{\text {lock }}$ was $7.5 \mathrm{~cm}$ lower and further along $z$ so as to practically eliminate RF crosstalk and couplings of discharge intensities between cells.

Pump laser - The laser source for the $1083 \mathrm{~nm}$ pump beam was a DBR diode laser (DL) with on-chip thermistor and thermoelectric cooler (Toptica LD-1082-0070-DBR) delivering $50 \mathrm{~mW}$ at our maximum operating current. The beam was collimated using an AR-coated aspheric lens of $8 \mathrm{~mm}$ focal length. It was linearly polarised, with a polarisation vector along $x$ (perpendicular to the plane of Fig. 4). The pump beam was mapped at the cell location $\left(0.2 \times 0.7 \mathrm{~cm}^{2} \mathrm{FWHM}\right)$ using a beam profiler (Thorlabs $\mathrm{BC} 106 \mathrm{~N})$. Its on-axis maximum intensity inside the cell $\left(\approx 90 \mathrm{~mW} / \mathrm{cm}^{2}\right)$ was fairly uniform over the area selected by the cropping diaphragm (D in Fig. 4). It was evaluated taking into account the reflectance of the cell windows (typ. $9 \%$ per window). The sample transmittance for various pump intensities was inferred from pairs of pump power measurements after the cropping diaphragm with RF discharge on and off.

In some experiments, the pump laser was used in freerunning operation, with its frequency set by the choice of the operating current and temperature (regulated using an in-house DL controller). To conveniently monitor pump absorption by the He gas in the cells, the discharge $\mathrm{RF}$ amplitudes could be modulated (around $80 \mathrm{~Hz}$ ). Corresponding lock-in demodulation of signals from $\mathrm{P}_{1}$ and $\mathrm{P}_{2}$ was then performed and outputs similar to the sketch in Fig. 3 p (see Fig. D.2 were obtained for $\mathrm{P}_{1}$ during pump frequency scans.

In other experiments, the pump laser frequency was locked on a helium transition using a standard saturated absorption scheme applied to gas in the ancillary cell $\mathrm{C}_{\text {lock }}$ (Fig. 4). A small modulation of the DL current at $1.5 \mathrm{kHz}$ was used to induce a pump frequency modulation, and lock-in demodulation provided a suitable error signal for a servo loop. The use of a quarter-wave plate allowed for convenient separation of the reflected beam by the $\mathrm{PC}-\mathrm{QW}$ element after a double pass in $\mathrm{C}_{\text {lock }}$. Moreover circularly polarised light yielded fewer (or single) line components in Zeeman spectra and therefore better frequency locking schemes. For most experiments with a locked pump laser, a mixture cell (with 1.07 mbar ${ }^{3} \mathrm{He}$ and 0.53 mbar ${ }^{4} \mathrm{He}$ ) was used to lock on any of the three ${ }^{4} \mathrm{He}$ lines or on the ${ }^{3} \mathrm{He} \mathrm{C}_{8}$ line.

Probe laser - The laser source for the $706.5 \mathrm{~nm}$ probe beam was an extended cavity diode laser (ECDL, Toptica DL $100 \mathrm{~L}$ ). We used a second in-house DL controller to manage the DL temperature and current, and computer control of the current and of the high voltage applied to the piezo actuator of the grating mount. Empirically determined joint linear variations of these current and voltage allowed for mode-hop-free tuning across the full helium spectra (> $40 \mathrm{GHz}$, see Fig. 2k). This range exceeds the nominal specifications $(21 \mathrm{GHz})$, which was sometimes convenient for wide frequency scans. However large amplitude noise was often observed near the boundaries of the scanned spectra, and such large frequency intervals involve large changes in the DL current, thus in the probe amplitude. Suitable DL temperature adjustments were therefore routinely used to centre the scanned intervals on regions of interest in the absorption spectra. For a DL current close to the maximum operating value at the lower frequency, the probe power decreased by about $30 \%$ at the higher frequency of typical $15 \mathrm{GHz}$ scans.

The probe wavelength was monitored and recorded during frequency scans using a Fizeau wavemeter (HighFinesse WS6-600). Its fibre input (not shown in Fig. 4) collected light deflected by the beam splitter $\mathrm{BS}_{2}$ in a horizontal plane.

Probe light detection - A general-purpose optical 
polarimeter (not shown in Fig. 4) included a polarising beam splitting cube (and an additional polariser in the deflected channel) for separation of the horizontal and vertical polarisation components of the probe light $(\mathrm{H}$ and $\mathrm{V}$, along $x$ and $y$ in Fig. 4 in the measurement cell $\mathrm{C}_{\text {exp }}$, respectively) 28, 29. The split beams were coupled by $f=30 \mathrm{~mm}$ lenses into $1-\mathrm{mm}$ core multimode plastic fibres that channelled light to Si PIN photodiodes (Siemens BPW34), and photocurrents were suitably converted to voltages. This light collecting scheme for the photodetectors $\mathrm{P}_{\mathrm{H}}$ and $\mathrm{P}_{\mathrm{V}}$ provided a high directional selectivity and made detection immune to stray light. It was used for the 1083-nm-sensitive detector $\mathrm{P}_{1}$ (Siemens BP104F) as well, whereas a large area photodiode (Centronic OSD50-4X) was directly placed on the path of the intense pump beam for $\mathrm{P}_{2}$.

The direction of the probe linear polarisation was rotated using a half-wave plate HW. Only horizontal or vertical polarisations in $\mathrm{C}_{\exp }$ (characterised by null signals from $\mathrm{P}_{\mathrm{V}}$ and $\mathrm{P}_{\mathrm{H}}$, respectively) were used in standard experiments. They were fully preserved by the dichroic mirrors $\mathrm{DM}_{1}$ and $\mathrm{DM}_{2}$ and were thus well defined in the experimental cell. Therefore, for a transverse applied magnetic field along the direction of the linear light polarisation ( $\pi$-polarised light), only $\Delta m=0$ transitions were excited or probed. Otherwise $\sigma$-polarised light was obtained for field directions perpendicular to that of the light polarisation (with equal intensities of $\sigma_{+}$and $\sigma_{-}$components), driving $\Delta m= \pm 1$ transitions. Combinations of $\pi$ and $\sigma$ polarisations, achieved for slanted orientations of the field in the tranverse plane, were used in some experiments.

Data acquisition - For a standard experiment, the pump frequency was fixed and locked to an atomic transition unless otherwise specified. The pump intensity was varied using attenuators. The pump intensity in the sample, and absorbed by the sample, were inferred from power measurements with discharge off and on (performed using the photodetector $\mathrm{P}_{2}$ at low pump intensities). For fixed discharge and pump settings, the probe laser frequency was linearly swept over a chosen interval, either in single pass or periodically for several scans. Probe frequency sweep rates of $200 \mathrm{MHz} / \mathrm{s}$ or slower were normally chosen, depending on the linewidths of the probed transitions.

During recordings the pump beam intensity was modulated (at $1.5 \mathrm{kHz}$ ) using a rotating wheel optical chopper. The signals from the photodetectors $\mathrm{P}_{\mathrm{H}}$ and $\mathrm{P}_{\mathrm{V}}$ were demodulated using digital lock-in amplifiers (Signal Recovery / EG\&G 7220). An output time constant of $20 \mathrm{~ms}$ (6dB/octave) was usually chosen. The raw photodetector signals were also integrated with simple RC networks to filter out the modulation, and both the integrated and the demodulated signals were digitally recorded. A 16bit USB data acquisition ADC module (Data Translation DT9816) with a 1-kHz sampling rate was managed by an in-house program which simultaneously controlled the probe laser frequency via a 12-bit DAC module (DT9813). Decimation of the oversampled data was performed in post-processing. Digital or aliased noise resulting from the digital data recording were thus made negligible. The variations of the probe wavelength (vacuum wavelengths versus time) were simultaneously recorded using the proprietary software of the wavemeter. The probe beam was transiently blocked at the beginning of each recording, which provided a time stamp used in data processing to accurately align optical and wavelength data and eliminate time.

\section{Results}

\subsection{Examples of double optical resonance spectra}

Illustrative examples of double optical resonance experimental spectra are compared in this section. The probe transmittance ${ }^{*} \mathcal{T}$, which can be directly linked with the atomic density distributions of velocities in the $2^{3} \mathrm{P}$ state sublevels (Secs. 2.2 and 2.3), was computed for each photodetector $\left(\mathrm{P}_{\mathrm{H}}\right.$ or $\left.\mathrm{P}_{\mathrm{V}}\right)$ from the ratio of demodulated signal magnitudes, $V_{\mathrm{RMS}}$, and averaged signal levels, $V_{\mathrm{av}}$ :

$$
{ }^{*} \mathcal{T}=\left[1-\beta V_{\mathrm{RMS}} / V_{\mathrm{av}}\right] /\left[1+\beta V_{\mathrm{RMS}} / V_{\mathrm{av}}\right] .
$$

This approach is reminiscent of that used in the case of weakly modulated densities of absorbers [22, App. A], but the density of $2^{3} \mathrm{P}$-state absorbers is here fully modulated with a near-square time pattern. For negligible rise and fall times of the pump intensity the coefficient in Eq. 30 would be $\beta=\pi /(2 \sqrt{2})$. The effect of the finite transition times resulting from the action of the pump beam chopper was assessed on the signals from the pump photodetector $\mathrm{P}_{2}$. As a result the corrected value $\beta=1.03 \pi /(2 \sqrt{2})$ was used.

In order to conveniently compare recorded transmittance spectra to theoretical expectations, frequency offsets (i.e. shifts from ${ }^{*} \mathrm{C}_{1}$ ) were inferred from wavelength measurements in two steps. First, recorded wavelength data were suitably processed to reduce spurious noise (as discussed in Sect.4.2) and interpolation of these processed values was used to ascribe a wavelength value to each computed transmittance value. Then the experimental frequency offset was obtained using our nominal offset (Table A.2 and the published vacuum wavelength ${ }^{3}{ }^{*} \lambda_{0}$ for the ${ }^{*} \mathrm{D}_{0}$ transition:

$$
\begin{aligned}
{ }^{*} \nu & ={ }^{*} \epsilon_{0}^{(4)} / h+c\left(1 /{ }^{*} \lambda-1 /{ }^{*} \lambda_{0}\right) \\
{ }^{*} \lambda_{0} & =706.76568 \mathrm{~nm} .
\end{aligned}
$$

\footnotetext{
${ }^{3}$ In the text we have used the traditional designation $706.5 \mathrm{~nm}$ for the transition wavelength, which refers to the mean air wavelength [12, 13, 14, 15, 16. The vacuum wavelength in Eq. 32 is indeed larger and can be obtained from the difference of level energies given in $\mathrm{cm}^{-1}$ in the NIST database [30 or in MHz in Ref. 31. Table 6]. The corresponding energy values in Ref. 24, Table 14] are slightly different, with a vacuum wavelength equal to $706.7657 \mathrm{~nm}$. This difference, corresponding to a $12 \mathrm{MHz}$ frequency shift, lies beyond the accuracy of our current wavelength measurements.
} 
Figure 5 displays examples of double optical resonance data selected to show the effect of the gas composition and pressure on the observed spectra. For the top row (a to
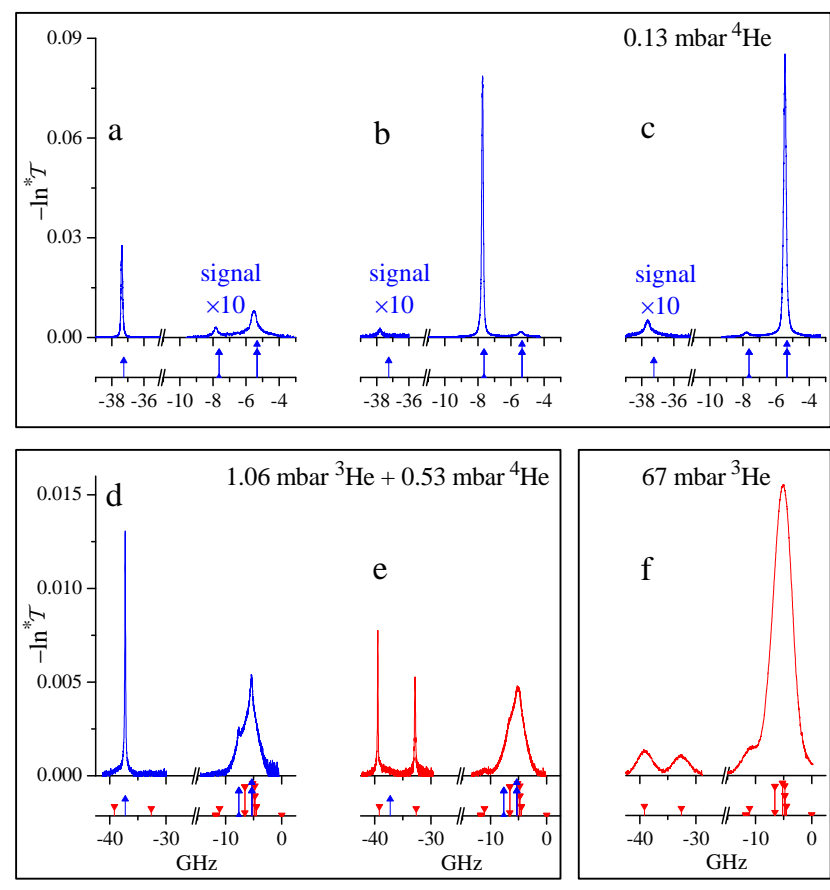

Figure 5: Examples of experimental double optical resonance spectra in low-pressure ${ }^{4} \mathrm{He}$ (a to c), an isotopic mixture ( $d$ and e), and high-pressure ${ }^{3} \mathrm{He}(\mathrm{f})$. The gas pressure is indicated in each box. The transmittance data are plotted vs. probe detuning from ${ }^{*} \mathrm{C}_{1}$. The pump frequency was tuned to $\mathrm{D}_{0}(\mathrm{a}), \mathrm{D}_{1}(\mathrm{~b})$, or $\mathrm{D}_{2}$ (c) for pure ${ }^{4} \mathrm{He} ; \mathrm{D}_{0}(\mathrm{~d})$ or $\mathrm{C}_{8}$ (e) for the mixture; $\mathrm{C}_{3}$ (f) for pure ${ }^{3} \mathrm{He}$. The on-axis pump intensity incident on the cell was $55 \mathrm{~mW} / \mathrm{cm}^{2}$ (a to c) or $0.1 \mathrm{~W} / \mathrm{cm}^{2}$ (d to f). Relevant transition matrix elements of Fig. 2 are displayed below each panel. Note the different transmittance and frequency scales for the top and bottom rows. The displayed frequency ranges add up to $12 \mathrm{GHz}$ (top) and $35 \mathrm{GHz}$ (bottom).

c), the pump selectively excited one of the three $J$-levels of the $2^{3} \mathrm{P}$ state of ${ }^{4} \mathrm{He}$. Absorption of the probe predominantly occurred from the pumped level, as expected. The weak absorption signals corresponding to atoms in the unpumped levels (note the 10-fold gain factor on one side of the broken axis in graphs a to c) result from small population transfers induced by $J$-changing collisions during the $2^{3} \mathrm{P}$ state radiative lifetime. For the experiments of Figs. $5 \mathrm{~d}$ and e, the $2^{3} \mathrm{P}_{0}$ levels of ${ }^{4} \mathrm{He}$ and ${ }^{3} \mathrm{He}$ were excited in the same cell using the well-resolved $\mathrm{D}_{0}$ and $\mathrm{C}_{8}$ pumping transitions, respectively. Narrow lines still correspond to absorption by atoms and isotopes selectively promoted to the pumped levels, and signals corresponding to absorption by atoms in the un-pumped levels are more salient than at lower pressure, as expected. With the large signal-to-noise ratio available in these measurements (the typical standard deviation in the baselines was
5 to $9 \times 10^{-5}$ ), the transition frequencies could be inferred with a small statistical uncertainty $(0.1-1 \mathrm{MHz})$. The agreement with the computed frequency offsets (depicted as bars in the graph below each spectrum) was usually fair but significant discrepancies (tens to hundreds of $\mathrm{MHz}$ ) were often found (for instance in graph $b$ for the main line and in graphs b and c for the weak lines). This issue is discussed in Sect. 4.2 and illustrated in Sect. 4.5

The high-pressure absorption data in Fig. 5 f show no evidence of narrow features, but resemble a computed Doppler-broadened spectrum (Fig. 2.). However, attempts (not shown) to fit absorption data using computed spectra revealed sizeable deviations in line shapes unless Voigt (rather than Gaussian) profiles were used, as expected, and a slightly weaker weight was ascribed to the $2^{3} \mathrm{P}_{0}$ population.

The total populations of the various levels of the $2^{3} \mathrm{P}$ state and population-transfer collision rates may be inferred from areas of the line components in such data. Globally, 80 to $90 \%$ of the $2^{3} \mathrm{P}$ population lied in the pumped state and velocity class for the low-pressure experiments (a to c). In contrast, only $25 \%$ remained in the pumped $2^{3} \mathrm{P}_{0}$ velocity class for the 1.6 mbar gas mixture ( $\mathrm{d}$ and e), and $10 \%$ (close to the thermalised limit of $1 / 9$ ) of the atoms were detected in the un-pumped $2{ }^{3} \mathrm{P}_{0}$ level for a 67 mbar ${ }^{3} \mathrm{He}$ gas submitted to excitation to the $2^{3} \mathrm{P}_{2}$ and $2^{3} \mathrm{P}_{x}$ levels (f).

The $B=0.16 \mathrm{mT}$ applied field was transverse and such that $B_{y}=2 B_{x}$ for the experiments on low-pressure ${ }^{4} \mathrm{He}$. The pump light had thus equal intensity components on each of the three light polarisation states $\left(\sigma_{+}, \sigma_{-}\right.$, and $\left.\pi\right)$, which efficiently prevented OP-induced build-up of atomic alignment in the $2^{3} \mathrm{~S}$ state. This yielded an increase in both pump and probe absorption signals by a factor $\sim 2$ compared to field orientations corresponding to a $\pi$ - or $\sigma$-polarised pump having the same total intensity. For experiments in cells containing a sizeable amount of ${ }^{3} \mathrm{He}$ (e.g., Figs. 5d to f), ME collisions with spin- $1 / 2$ ground state atoms efficiently prevented alignment to occur in the $2^{3} \mathrm{~S}$ state, and absorption of pump and probe were indeed observed to be almost unaffected by the direction of the applied field.

\subsection{Probe wavelength measurements}

The absolute accuracy of our wavemeter was $600 \mathrm{MHz}$, but its specified resolution $(20 \mathrm{MHz})$ and its readout resolution $\left(10^{-5} \mathrm{~nm}\right.$, hence $6 \mathrm{MHz}$ at the probe wavelength) could yield fairly precise measurements. This feature of wavemeters based on Fizeau interferometers has been applied by several groups to precisely lock lasers at fixed frequencies using devices from the same manufacturer that have the highest available accuracy $(10 \mathrm{MHz})[32,33$. We have evaluated the time stability of measurement errors by monitoring readout frequency drifts at fixed laser frequencies, for the un-swept probe laser sitting on a narrow double optical resonance line, or for the locked pump laser. 
Slow readout frequency drifts were observed in all cases, and rapid changes were observed when the wavemeter input fibre was bent or moved and when the light injection into the fibre was geometrically modified (see an example in Fig. D.1 in Appendix D). The frequencies indeed remained within the instrument accuracy, and in stable conditions the frequency drifts did not exceed $10 \mathrm{MHz}$ during the time needed for a frequency scan across a linewidth $(\sim 1 \mathrm{~s})$ or a line splitting interval of interest (10 to $30 \mathrm{~s})$. In spite of this fair time stability of the readout error, recordings of readout frequencies for swept lasers (both the pump and the probe) indicated that this instrumental error depended on the measured wavelength.

Figures $6 \mathrm{a}$ and b display examples of deviations of the wavemeter readout values from expected linear variations with the pump laser frequency control parameter.Most
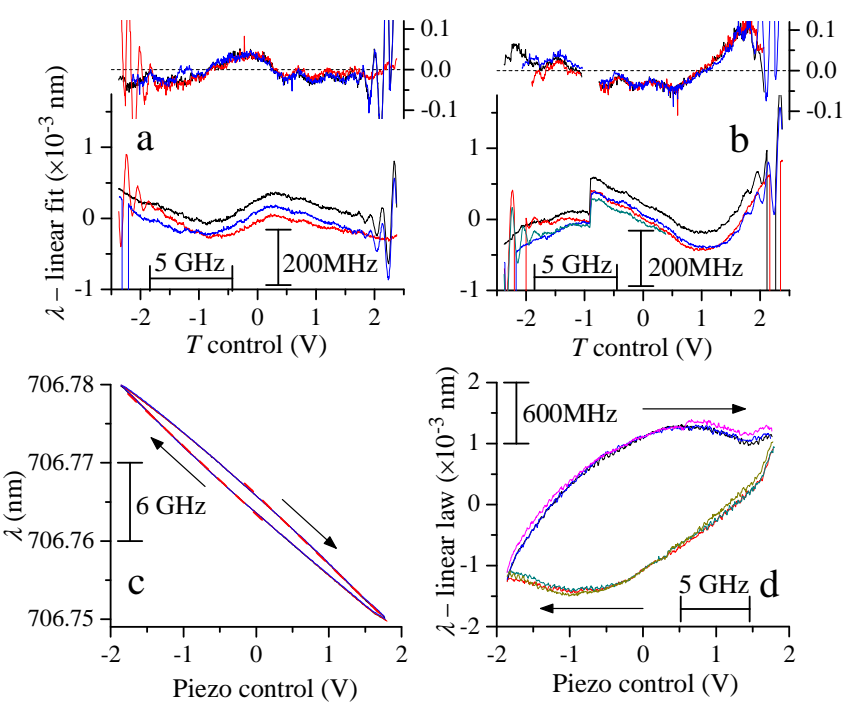

Figure 6: Plots of processed data obtained from wavemeter readout values $\lambda$ vs. the laser frequency control parameters (repeated back and forth scans appear in different colours). a,b: Pump laser at 1083 nm (vs the DL temperature control parameter). Lower traces: examples of deviations of $\lambda$ from linear fits, with slopes $s_{\lambda}$, for the pump laser; Upper traces: derivatives of these deviations scaled to $s_{\lambda}$. c,d: Probe laser at $706.5 \mathrm{~nm}$. c: Example of $\lambda$ variation with the piezo actuator control for the probe laser. d: Corresponding differences to a linear law with the common slopes fit to the second halves of the hysteresis loops in c. The arrows in $\mathrm{c}$ and $\mathrm{d}$ indicate increasing times. Scale conversions to frequency units are displayed.

recordings exhibit smooth variations (Fig. 6a) but apparent jumps were sometimes observed (Fig. 6p). The damped oscillations following each turning point in the triangular sweeps were actual DL temperature (and thus wavelength) oscillations but the other features were variations in the wavemeter error similar to those recently reported for a high-accuracy device [34]. The use of wavemeter data instead of the true wavelengths for probe absorption data processing therefore induced a local scaling error impacting for instance the obtained line widths or areas. This scaling error is given by the reduced derivatives of the deviations, which are plotted as the upper traces in each graph and typically did not exceed $\pm 5 \%$.

When the probe laser wavelength was recorded a clear hysteretic response was observed (Fig. 6.). Subtracting a linear function of the control parameter (Fig. 6d) did not suggest a reliable parametrisation for this behaviour, which was attributed to the mechanical response of the piezo actuator. We could therefore not avoid the local scaling errors resulting from the use of wavemeter data, and simply assumed that they had similar magnitudes at pump and probe wavelengths. These frequencydependent errors were usually stable for successive recordings, but lost their correlation for recordings made hours or days apart (as in Figs. 6a and b). Averaging results of such distant measurements may therefore be used to reduce the effect of the frequency-dependent readout errors on the line width and line splitting measurements.

\subsection{Examples of Zeeman-split spectra}

Figure 7 displays probe absorption spectra obtained in an applied longitudinal field when the $2^{3} \mathrm{P}_{0}$ state of ${ }^{3} \mathrm{He}$ (a) or ${ }^{4} \mathrm{He}$ (b) was pumped. The Zeeman shifts derived in Sect. 2.4 were used to compute the expected line positions represented below the spectra.

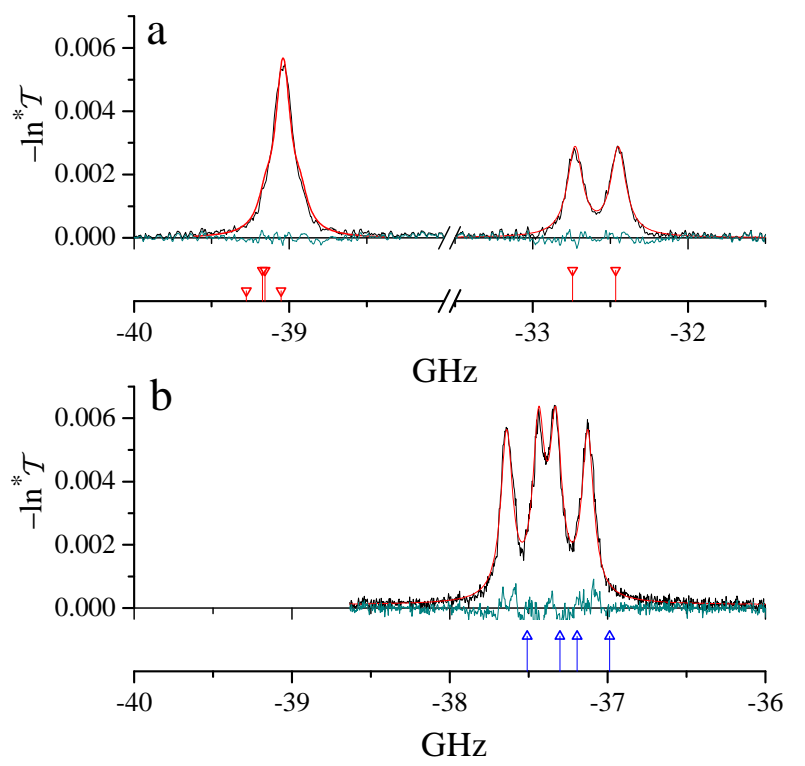

Figure 7: Double optical resonance spectra displaying combined Zeeman line splittings ( $\sigma$-polarised pump and probe beams). a: Pump tuned to $\mathrm{C}_{8}\left(55 \mathrm{~mW} / \mathrm{cm}^{2}\right), B_{z}=$ $3.14 \mathrm{mT}, 0.27+0.27$ mbar isotopic mixture. b: Pump tuned to $\mathrm{D}_{0}\left(100 \mathrm{~mW} / \mathrm{cm}^{2}\right), B_{z}=3.68 \mathrm{mT}, 0.133 \mathrm{mbar}$ ${ }^{4}$ He gas. Experimental data (black), Lorentzian fits (red, Eqs. 33 to 35, and residues (teal) are displayed. The bars below the spectra are the computed positions and transition matrix elements of the line components. Note the 4.5-GHz-long break in the frequency axis of plot a. 
The ${ }^{3} \mathrm{He}$ line component shifts were obtained using Eq. 27. For $\mathrm{C}_{8}$ pump and ${ }^{*} \mathrm{C}_{8}$ probe all $F$-values are equal to $1 / 2$ and only two excitation paths $\hat{m}_{1} \rightarrow$ $\hat{m}_{2} \rightarrow \hat{m}_{3}$ are allowed for $\sigma$-polarised light: $\pm 1 / 2 \rightarrow$ $\mp 1 / 2 \rightarrow \pm 1 / 2$, yielding a doublet with shift factors $\pm \beta_{8}= \pm 44.18 \mathrm{MHz} / \mathrm{mT}$ (Eq 28). For the ${ }^{*} \mathrm{C}_{9}$ probe, $F=$ $3 / 2$ in the upper level and four excitation paths are allowed, yielding a barely split strong doublet $\left(\hat{m}_{3}= \pm 3 / 2\right.$, $\left.\pm \beta_{9,3}= \pm 2.54 \mathrm{MHz} / \mathrm{mT},{ }^{*} T_{17,4}=0.0937\right)$ and a weak doublet $\left(\hat{m}_{3}= \pm 1 / 2, \pm \beta_{9,1}= \pm 34.84 \mathrm{MHz} / \mathrm{mT},{ }^{*} T_{17,2}=\right.$ $\left.{ }^{*} T_{17,4} / 3\right)$. The recorded spectra exhibit the corresponding features in Fig. 7 a, and sums of Lorentzian profiles (the red lines) accurately reproduce the experimental data. Four parameters were used in the fitting function $\mathcal{F}_{8}$ for ${ }^{*} \mathrm{C}_{8}$ spectra, yielding values for the total line area $A_{8 \mathrm{fit}}$, the common width $W_{8 \mathrm{fit}}$, the field strength $B_{8 \mathrm{fit}}$, and a frequency offset $\delta_{8 \text { fit }}$.

$$
\begin{aligned}
\mathcal{F}_{8}\left(\nu_{0}\right) & =A_{8 \mathrm{fit}}\left[\mathcal{L}_{W 8 \mathrm{fit}}\left(\nu_{0}-{ }^{*} \nu_{8}+\delta_{8 \mathrm{fit}}+\beta_{8} B_{8 \mathrm{fit}}\right)\right. \\
& \left.+\mathcal{L}_{W 8 \mathrm{fit}}\left(\nu_{0}-{ }^{*} \nu_{8}+\delta_{8 \mathrm{fit}}-\beta_{8} B_{8 \mathrm{fit}}\right)\right] / 2
\end{aligned}
$$

The fit value $B_{8 \text { fit }}$ was found in good agreement with $B_{z}$, the $0.5 \%$ difference being lower than the possible scaling errors in frequency scale discussed in Sect. 4.2. Three parameters were used in the fitting function $\mathcal{F}_{9}$ for ${ }^{*} \mathrm{C}_{9}$ spectra: $A_{9 \mathrm{fit}}, W_{9 \mathrm{fit}}$, and $\delta_{9 \mathrm{fit}}$, while the applied field $B_{z}$ was used since the poorly resolved lines could not reliably yield both field and width values.

$$
\begin{aligned}
\mathcal{F}_{9}\left(\nu_{0}\right) & =A_{9 \text { fit }}\left[3 \mathcal{L}_{W 9 \text { fit }}\left(\nu_{0}-{ }^{*} \nu_{9}+\delta_{9 f i t}+\beta_{9,3} B_{z}\right)\right. \\
& +3 \mathcal{L}_{W 9 \text { fit }}\left(\nu_{0}-{ }^{*} \nu_{9}+\delta_{9 \mathrm{fit}}-\beta_{9,3} B_{z}\right) \\
& +\mathcal{L}_{W 9 \text { fit }}\left(\nu_{0}-{ }^{*} \nu_{9}+\delta_{9 \mathrm{fit}}+\beta_{9,1} B_{z}\right) \\
& \left.+\mathcal{L}_{W 9 \text { fit }}\left(\nu_{0}-{ }^{*} \nu_{9}+\delta_{9 \mathrm{fit}}-\beta_{9,1} B_{z}\right)\right] / 8 .
\end{aligned}
$$

The frequency offsets $\delta_{8 \mathrm{fit}}=17 \mathrm{MHz}$ and $\delta_{9 \mathrm{fit}}=124 \mathrm{MHz}$ both lied within the precision of the frequency measurements $(600 \mathrm{MHz})$. They differed from a common expected offset associated with the use of circularly polarised light to set or lock the pump laser frequency (Fig. 4), and such difference is discussed in more detail in Sect. 4.5. The linewidths $W_{8 \text { fit }}=119 \mathrm{MHz}$ and $W_{9 \text { fit }}=115 \mathrm{MHz}$, each with a $2 \mathrm{MHz}$ statistical uncertainty and a few $\%$ of uncertainty arising from possible scaling errors are in satisfactory agreement. Finally, the ratio $A_{9 \mathrm{fit}} / A_{8 \mathrm{fit}}=1.262$ of the line areas parameters was found to be consistent with the expected value, $\left({ }^{*} T_{17,4}+{ }^{*} T_{17,2}\right) /{ }^{*} T_{17,5}=1.246$.

The ${ }^{4} \mathrm{He}$ shifts of Fig. $7 \mathrm{~b}$ were obtained using Eq. 28. Four double resonance excitation paths through $m_{J}=0$ are allowed for $\sigma$-polarised light: $m_{S}= \pm 1$ to ${ }^{*} m_{S}= \pm 1$, yielding one doublet with shift factors $\pm \beta_{0 l}= \pm 70.91 \mathrm{MHz} / \mathrm{mT}$, and $m_{S}= \pm 1$ to ${ }^{*} m_{S}=\mp 1$, yielding another doublet with shift factors $\pm \beta_{0 s}= \pm 14.91 \mathrm{MHz} / \mathrm{mT}$. A fitting function $\mathcal{F}_{0}$ for ${ }^{*} \mathrm{D}_{0}$ with parameters $A_{0 \text { fit }}, W_{0 \text { fit }}, B_{0 \text { fit }}$, and $\delta_{0 \text { fit }}$ was used to model the Zeeman-split line in Fig. $7 \mathrm{~b}$

$$
\begin{aligned}
& \mathcal{F}_{0}\left(\nu_{0}\right)=A_{0 \text { fit }}\left[\mathcal{L}_{W 0 \text { fit }}\left(\nu_{0}-{ }^{*} \nu_{0}^{(4)}+\delta_{0 \text { fit }}+\beta_{0 l} B_{0 \text { fit }}\right)\right. \\
& +\mathcal{L}_{W 0 \mathrm{fit}}\left(\nu_{0}-{ }^{*} \nu_{0}^{(4)}+\delta_{0 \mathrm{fit}}-\beta_{0 l} B_{0 \mathrm{fit}}\right) \\
& +\mathcal{L}_{W 0 \mathrm{fit}}\left(\nu_{0}-{ }^{*} \nu_{0}^{(4)}+\delta_{0 \mathrm{fit}}+\beta_{0 s} B_{0 \mathrm{fit}}\right) \\
& \left.+\mathcal{L}_{W 0 \mathrm{fit}}\left(\nu_{0}-{ }^{*} \nu_{0}^{(4)}+\delta_{0 \mathrm{fit}}-\beta_{0 s} B_{0 \mathrm{fit}}\right)\right] / 4 \text {. }
\end{aligned}
$$

Again, the fit value $B_{0 \text { fit }}\left(1.5 \%\right.$ lower than $\left.B_{z}\right)$ and the frequency offset $\delta_{0 \text { fit }}=-138 \mathrm{MHz}$ were in line with expectations. The linewidth $W_{\text {0fit }}=90 \mathrm{MHz}$, which was obtained with a $1 \mathrm{MHz}$ statistical uncertainty, cannot be directly compared at this stage with that obtained at a different pressure, for the other isotope, and with a different pump intensity. We still notice that, for the two ${ }^{3} \mathrm{~S}-2^{3} \mathrm{P}_{0}$ pumping schemes considered here, two narrow velocity classes with identical widths can be observed for the atoms detected in the $2^{3} \mathrm{P}_{0}$ level (one class for each of the two magnetic sublevels in ${ }^{3} \mathrm{He}$, two classes with opposite mean velocities in ${ }^{4} \mathrm{He}$ when the pump is tuned to the resonant atomic frequency for $B=0$ ). Therefore a single width parameter can be used in all fit functions, which yields robust fit results even if the Zeeman components are ill-resolved. We therefore have a suitable tool for fits of narrow (Doppler-free) lines, from which areas and widths can be reliably extracted. The situation would be more complex for pumping schemes addressing different sublevels with different pumping rates, for instance.

\subsection{Effect of pump intensity}

The influence of pump light intensity on probe absorption signals was studied for several conditions of gas composition and pressure, as well as pump light polarisation and frequency.

The strength of probe absorption was characterised by the areas of the narrow spectral lines corresponding to directly pumped atomic sublevels in each experiment. The areas were usually obtained from fit parameters of suitable model functions. They were checked to be consistent with those obtained from numerical integration whenever spectral shapes and signal-to-noise ratio allowed for it, for instance at high pump intensity and low pressure. Figure 8 a displays data obtained in different cells for $\pi$-polarised pump and probe light, plotted as functions of average pump intensities $\left\langle I_{\mathrm{P}}\right\rangle$ (which were computed assuming exponential decay along the pump path). A wide range of values was obtained at fixed pump intensity, as could be noticed for instance in Fig. 5. The solid lines are lowintensity areas of Eq. 21 computed for the experiments made in the low-pressure cell using the low-intensity transmittance $\mathcal{T}_{0}$. They agree with the corresponding data (triangles) within $10 \%$ below a few $\mathrm{mW} / \mathrm{cm}^{2}$, and the differences at higher intensities emphasize the strong loss of efficiency in populating the probed $2^{3} \mathrm{P}$ state over most of the pump intensity range. All area values tend to saturate at large pump intensity, which correlates with the 
decrease of pump absorption plotted in Fig. $8 \mathrm{p}$ for the corresponding experiments. It is worth noticing that the decrease with $I_{\mathrm{P}}$ was faster for pure ${ }^{4} \mathrm{He}$ (all solid symbols), in which atomic alignment was produced in the $2^{3} \mathrm{~S}$ state by $\pi$-polarised pump light. This optical pumping effect reduced absorption in that case, but it was efficiently hindered by ME collisions when ${ }^{3} \mathrm{He}$ or isotopic gas mixtures were used. Of course, enforcing equal intensities of the three pump light polarisation components (see Sect. 4.1) was another way to avoid optical pumping effects and to enhance absorption at large $I_{\mathrm{P}}$ in pure ${ }^{4} \mathrm{He}$.
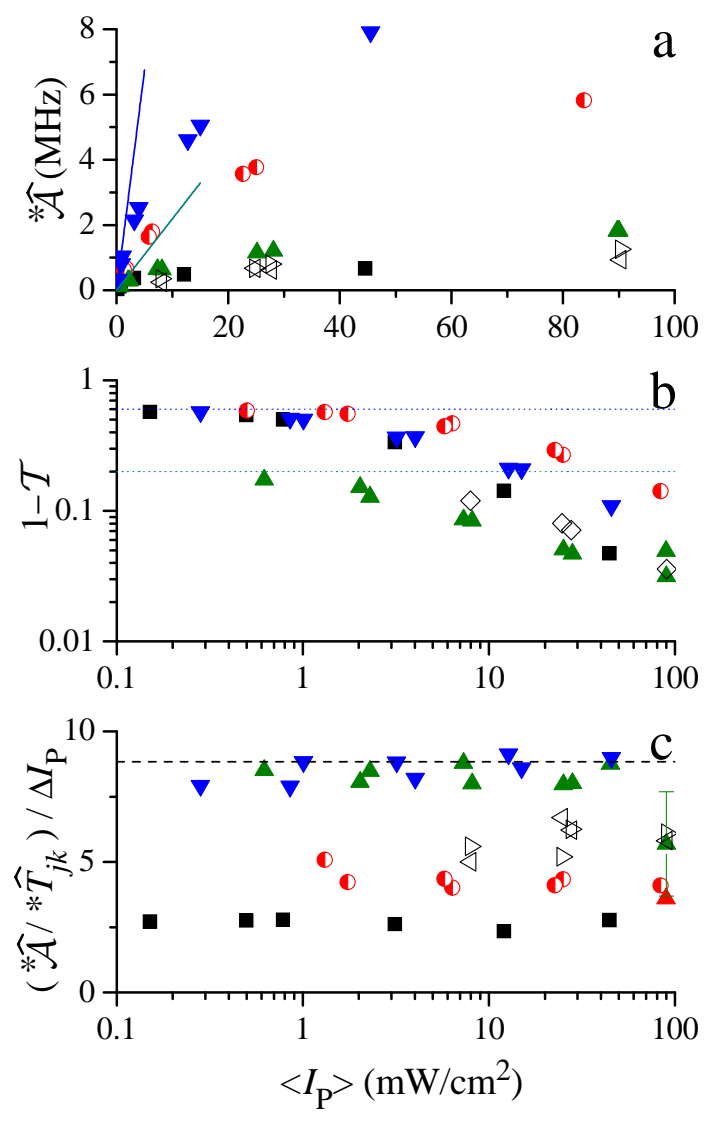

Figure 8: Series of data plotted vs. $z$-averaged pump intensities, for different gas samples and pumping lines: ${ }^{4} \mathrm{He}$ at $0.13 \operatorname{mbar}\left(\boldsymbol{\nabla}: \mathrm{D}_{1}, \boldsymbol{\Delta}: \mathrm{D}_{0}\right)$ and $1 \operatorname{mbar}\left(\mathbf{\square}: \mathrm{D}_{0}\right) ;{ }^{3} \mathrm{He}$ at 0.4 mbar $\left(\mathrm{C}_{8}\right.$ pump, $\triangleright:{ }^{*} \mathrm{C}_{8}$ probe, $\triangleleft:{ }^{*} \mathrm{C}_{9}$ probe $) ;{ }^{4} \mathrm{He}$ at $0.4 \mathrm{mbar}+{ }^{3} \mathrm{He}$ at $0.13 \mathrm{mbar}\left(\mathrm{O}_{0} \mathrm{D}_{0}\right)$. Plot a: Areas of narrow absorption lines corresponding to directly pumped $2^{3} \mathrm{P}$ levels. The lines are computed areas for weak $\mathrm{D}_{1}$ and $\mathrm{D}_{0}$ pumps in the 0.13 mbar cell (Eq. 21 assuming $\mathcal{R}=1$ ). b: Absorbed fraction of the pump intensity $\left(\diamond:{ }^{3} \mathrm{He}\right.$ at 0.4 mbar). The dotted lines correspond to $\mathcal{T}_{0}$ values in the 0.13 mbar cell for very small $I_{\mathrm{P}}$. c: Scaled ratios of Eq. 18. The dashed line is the value of Eq. 19. The choice of linear or logarithmic scales was made for clarity of presentation.

Figure 8c combines the results obtained for measured areas (data of Fig. 8a) and the measured pump intensity differences (between input and output of the gas samples),
$\Delta I_{\mathrm{P}}$, as suggested by Eq. 18 . The experimental errors for the areas mainly arose from frequency scaling errors (up to $\pm 5 \%$ ) discussed in Sect. 4.2. For the weakest absorptions at high $I_{\mathrm{P}}$ the accuracy on $\Delta I_{\mathrm{P}}$ was rather poor as a result of large interference from the $\mathrm{RF}$ discharge (see a corresponding worst-case error bar in Fig. 8k). Allowing for these errors, the computed ratios were found to be quite independent of pump intensity for each sample, as expected. Moreover, their values were consistent with the expectation of Eq. 18, with a fraction $\mathcal{R}$ of atoms close to 1 at 0.13 mbar and decreasing with increasing pressure and collision rates $\left(\mathcal{R}\right.$ is the fraction of atoms in the $2^{3} \mathrm{P}$ state which have remained in the pumped sublevel(s) and velocity class before radiative decay).

Investigations of optical pumping effects in the $2^{3} \mathrm{~S}$ state and of their consequences on the total populations in the $2^{3} \mathrm{P}$ state could indeed only involve pump absorption measurements, but $706.5 \mathrm{~nm}$ probe absorption measurements may yield higher accuracy especially in low-pressure samples. Moreover they provide additional information when the lineshapes and widths are analysed, in addition to the line areas. A systematic compilation of the spectral data obtained in our experiments was performed. An overall increase of linewidths with gas pressure and a slow increase with pump intensity were observed. However, a sofar unexplained systematic deviation from the expected simple Lorentzian lineshape was observed in low-pressure gas samples. Further investigations are needed before a comprehensive analysis can be reported.

\subsection{Feasibility of Doppler-free spec- troscopy}

Following the conclusion of Sect. 4.2, series of repeated scans of the $2^{3} \mathrm{P}_{0}-3^{3} \mathrm{~S}$ transitions in isotopic gas mixtures were performed with the pump frequency locked to the $\mathrm{C}_{8}$ or the $\mathrm{D}_{0}$ line. The experiments were performed in null field to avoid Zeeman line shifts. Indeed, with linear pump and probe polarisations, Zeeman splittings (such as observed in Fig. 7) were not expected to affect centre-ofline positions. In contrast, the saturated absorption beam was circularly polarised and therefore the pump frequency would be Zeeman-shifted proportionally to any $B_{z}$ field. The three Earth's field components were compensated and in particular $\left|B_{z}\right|<2 \mu \mathrm{T}$, resulting in a worst-case frequency shift below $0.1 \mathrm{MHz}$ in these experiments (Eq. 28).

Figure 9a displays the time variations of the probe wavelength and absorption spectra for six back and forth scans, for a $\mathrm{C}_{8}$-tuned pump. For each scan in a recording the ${ }^{*} \mathrm{C}_{8}$ and ${ }^{*} \mathrm{C}_{9}$ absorption lines were fit using Lorentzian profiles, yielding sets of fit frequencies, linewidths, and statistical uncertainties.

Fig. 9b displays the fit frequencies of the ${ }^{*} \mathrm{C}_{8}$ transition in Fig. 9a together with those from three more recordings made on the same day. The frequency drifts between scans of a recording, or between recordings, were on the same order of magnitude as the typical linewidth represented 

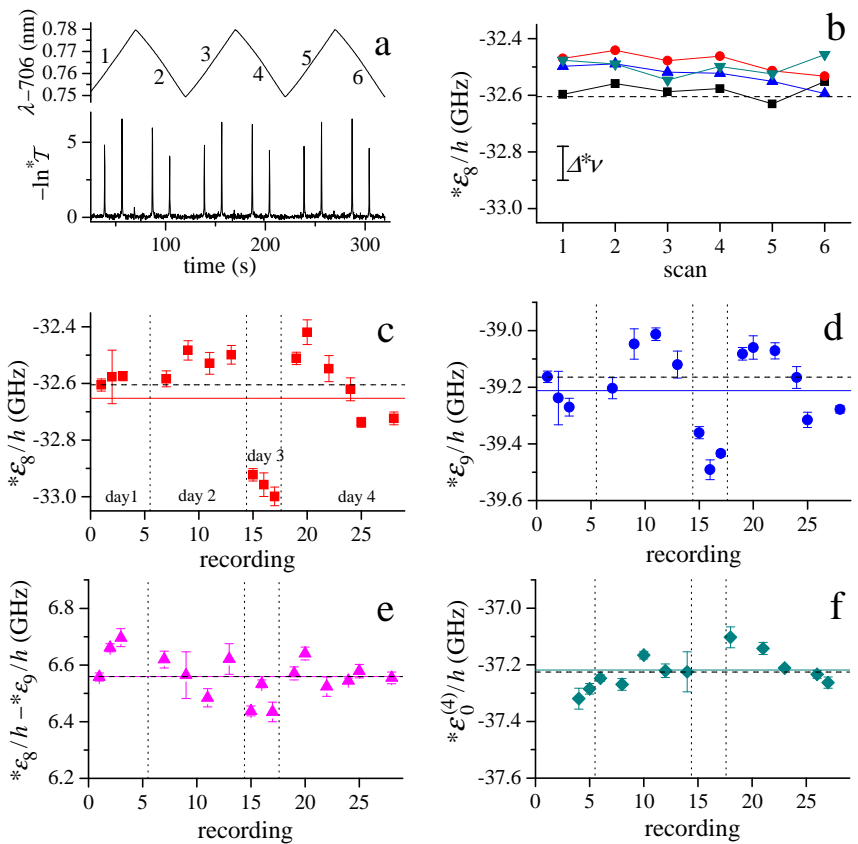

Figure 9: Results of series of repeated scans across the $2^{3} \mathrm{P}_{0}-3^{3} \mathrm{~S}$ absorption lines in isotopic mixtures with a pumped $2^{3} \mathrm{P}_{0}$ level. a: Typical recording comprising six scans for a $\mathrm{C}_{8}$-tuned pump. b: Fit frequencies of the lines in a set of recordings are plotted vs. the scan number (squares: data from Fig. a; other symbols: three more recordings on the same day; the lines connect values of one recording). c, d, f: The averages (symbols) and standard deviations (error bars) of the six scans were derived for each recording. $c$ and $d: C_{8}$ pumping, $f: D_{0}$ pumping. e: Difference of the data in $\mathrm{c}$ and $\mathrm{d}$, yielding the hyperfine splitting in the $3^{3} \mathrm{~S}$ state of ${ }^{3} \mathrm{He}$. The solid lines are the averages of all data, the dotted lines are the computed values (Table A.2.

in Fig. 9b $\left(\Delta^{*} \nu=120 \mathrm{MHz}\right)$. The statistical uncertainty on the fit frequencies $(\sim 1 \mathrm{MHz})$ was smaller than the symbol sizes.

The results of all measurements for the ${ }^{*} \mathrm{C}_{8}$ transition are compiled in Fig. 9r, in which the symbol positions and error bars stand for the averages and standard deviations of fit values obtained in a recording: the four recordings used in Fig. 9p yield the data points of day 2. The results in Figs. 9k to 9 e correspond to 29 recordings made on four different days using two cells with different gas mixtures: $0.27+0.27$ mbar ${ }^{3} \mathrm{He}+{ }^{4} \mathrm{He}$ on consecutive days 1 and 2, and $0.13+0.4$ mbar on days 3 and 4 one week later. The pump was tuned to $\mathrm{C}_{8}$ to excite ${ }^{3} \mathrm{He}$ atoms (Figs. 9 to 9e, 17 recordings) or to $\mathrm{D}_{0}$ to excite ${ }^{4} \mathrm{He}$ atoms (Fig. 9f, 12 recordings).

Using similar plotting conventions Fig. 9e displays the differences in fit frequency for the ${ }^{*} \mathrm{C}_{8}$ and ${ }^{*} \mathrm{C}_{9}$ lines computed for each scan recorded with $\mathrm{C}_{8}$ pumping. The inrecording standard deviations (the error bars in Fig. 9p) and the scatter of average values of the line splittings are somewhat reduced compared to the absolute frequency measurements, but the frequency-dependent wavemeter errors were not eliminated in the process.

Table 1 lists the quantitative results of this series of measurements in terms of deviations from the computed values. The values found for the hyperfine splitting in the $3^{3} \mathrm{~S}$ state of ${ }^{3} \mathrm{He}$ and for the isotopic shift of the $706.5 \mathrm{~nm}$ transition are in good agreement with expectations in spite of the large and frequency-dependent readout errors of the wavemeter used in these experiments which makes this instrument ill-suited for accurate spectroscopic measurements. The standard errors obtained for repeated measurements suggest that the measurement errors were in fact efficiently averaged.

\begin{tabular}{c|c|c|c|c}
$\begin{array}{c}\text { line } \\
\text { name }\end{array}$ & $\begin{array}{c}\text { av. }- \text { th. } \\
(\mathrm{MHz})\end{array}$ & $\begin{array}{c}\mathrm{SD} \\
(\mathrm{MHz})\end{array}$ & $\begin{array}{c}\mathrm{SE} \\
(\mathrm{MHz})\end{array}$ & $N_{\mathrm{s}}$ \\
\hline${ }^{*} \mathrm{C}_{8}$ & -38.1 & 177 & 18.5 & 92 \\
${ }^{*} \mathrm{C}_{9}$ & -39.7 & 144 & 15 & 92 \\
${ }^{*} \mathrm{D}_{0}$ & 2.0 & 63.6 & 7.5 & 72 \\
\hline \hline${ }^{*} \mathrm{C}_{9}-{ }^{*} \mathrm{C}_{8}$ & 1.6 & 76.5 & 8 & 92
\end{tabular}

Table 1: Statistical data for the compiled recordings of the three lines probed in the experiments of Fig. 9. Columns 2 to 4 contain the values of the differences between the averages of the fit frequencies and the computed values, the standard deviation and the standard error of the fit frequencies. $N_{\mathrm{s}}$ is the number of scans used for these averages. The last line contains the data for the ${ }^{3} \mathrm{He}$ hyperfine splitting measurements (see text and Fig. 9e)

\section{Discussion}

Calculations of the level structures and optical transition details for the $1083 \mathrm{~nm}$ transition of Ref. [17] were extended to the $706.5 \mathrm{~nm}$ transition. Low-field explicit expressions of Zeeman shifts of the $2^{3} \mathrm{~S}, 2^{3} \mathrm{P}$, and $3^{3} \mathrm{~S}$ levels involved in double optical resonance are given in this article. This extension is indeed valid for arbitrary magnetic field using straightforward changes in the numerical computations of Ref. [17]. Regarding double optical resonance schemes, quantitative links were derived between integrated probe absorption spectra at $706.5 \mathrm{~nm}$ and atomic densities in the $2^{3} \mathrm{P}$ state or pump absorption.

As regards lineshapes, the low-pump-intensity limit was derived and the power broadening effects on velocity distributions in the $2^{3} \mathrm{~S}$ and $2^{3} \mathrm{P}$ states were briefly discussed in Sect. 2.3. This would indeed automatically induce a related broadening of probe absorption lines. Additional broadening of the probe lines resulting from an intense pump may be expected for this 3-level ladder-type excitation scheme. Various theoretical approaches have been used in similar situations (e.g., 35, 36, 37]). A pumpinduced increase of the damping rate of the probe transition may be expected, with changes in the lineshapes ex- 
pected from Rabi oscillations driven by an intense pump in a dressed-atom approach. Such effects are not believed to play a significant role in our experiments where strong optical saturation is not achieved, but a dedicated study at higher pump light intensity would probably benefit from the use of optical probes on both optical transitions.

A simple experimental scheme was used with collinear pump and probe beams combined using dichroic mirrors. This restricted the beam light polarisations to being linear and either $s$ - or $p$-polarised (horizontal or vertical polarisations in our setup). This was required to avoid strong modifications of the beam polarisations, which are well known for reflections on dichroic mirrors 38 and were observed for the transmitted beam as well. Experiments requiring arbitrary beam polarisations could advantageously be performed with pump and probe beams propagating with a small angle to avoid complex compensation of the effects of dichroic mirrors, at the expense of a loss of compactness of the system.

Double optical resonance spectra recorded with our simple scheme over a wide range of pressures displayed very clear features, with narrow Doppler-free lines below a few mbar. In that case complex Zeeman splittings resulting from the interplay of the Zeeman shifts and pump-induced velocity selection were observed and found to agree well with expectations. This provides a basis for reliable analyses and fits of observed spectra in applied magnetic fields, in which line shapes, widths, and positions of individual components can be reliably determined even in poorly resolved Zeeman structures. Conversely, above a few tens of mbar, broad thermal spectra provide evidence of efficient velocity-changing collisions during the radiative lifetime of the $2^{3} \mathrm{P}$ state; the velocity-selective character of the pump is essentially lost but the tremendous increase in signal-to-noise ratio of this approach, compared to conventional absorption or fluorescence techniques, still makes our pump-probe scheme very useful. And indeed, for studies in the intermediate pressure regime which have motivated these experiments, analyses of recorded spectra for suitable combinations of pump and probe frequencies and polarisations can be expected to provide a wealth of useful data. The reported observations of the influence of pump intensity on signal amplitudes already highlight the pressure dependence of the fractional transfer of populations, both in pure ${ }^{4} \mathrm{He}$ and isotopic mixtures. It also provides values for suitable pump intensities in various situations, with large enough probe absorption and moderate saturation broadening.

Finally, an intrinsic frequency-dependent measurement error of a Fizeau-type wavemeter was evidenced and characterized. The resulting limitation on the precision of spectroscopic measurements on the Doppler-free $706.5 \mathrm{~nm}$ transition was evaluated, yielding a 10 to $20 \mathrm{MHz}$ statistical error on line positions. Obtaining spectroscopically useful results, with accuracies not exceeding sub-MHz statistical errors in a single scan, would require a more accurate wavelength measurement instrument or method in order to settle, for instance, the $0.55 \mathrm{MHz}$ discrepancy between the published isotopic shift differences of Eqs. A.6 and A.7. Suitable measurements could be performed using, for instance, a more accurate model by the same manufacturer (with a 60-fold smaller stated error), or a stable interferometer of the kind used in Ref. 39 for the determination of the isotope shift between line components of the $1083 \mathrm{~nm}$ transition.

\section{Acknowledgements}

We thank V. Fossati for contributions to exploratory steps of the experiments and A. Lemettre for enhancements of the $B$-field coils, performed in the frame of independent internships.

\section{Authors contributions}

G.T and P.J.N. designed and initiated the project. A.D. performed most of the reported experimental work and data analysis. P.J.N. performed the calculations and wrote the manuscript with input from all authors.

\section{Appendix}

\section{A Computed level and optical transition data}

The upper part of Table A.1 lists the null-field ${ }^{3} \mathrm{He}$ energy terms (in units of frequency) of the different hyperfine levels computed using the effective Hamiltonian of Ref. 17. The difference between the $2^{3} \mathrm{~S}$ and $3^{3} \mathrm{~S}$ level energy terms results from the values of their hyperfine structure constants [31, Table 7]

$$
\begin{aligned}
& A_{\mathrm{S}}\left(2^{3} \mathrm{~S}\right)=4493.134 \mathrm{MHz} \\
& A_{\mathrm{S}}\left(3^{3} \mathrm{~S}\right)=4372.893 \mathrm{MHz} .
\end{aligned}
$$

The computed low-field variations of the energy terms with field $B$ for all Zeeman sublevels were used to extract the linear coefficients, expressed for each of the nine levels listed in Table A.1 as:

$$
\hat{E}(B)-\hat{E}(0)=\hat{g}_{F} \hat{m}_{F} \mu_{B} B+\mathcal{O}\left(B^{2}\right)
$$

where $\mu_{\mathrm{B}}$ is the Bohr magneton, $\hat{E}, \hat{g}_{F}$, and $\hat{m}_{F}$ are generic notations for the energy, Landé factor, and angular momentum projection of a ${ }^{3} \mathrm{He}$ sublevel. The lower part of Table A.1 lists the null-field energy terms of the three $2^{3} \mathrm{P}_{J}$ levels of ${ }^{4} \mathrm{He}$. Their low-field variations is

$$
E_{\mathrm{P}}^{(4)}(B)-E_{\mathrm{P}}^{(4)}(0)=g_{J} m_{J} \mu_{B} B+\mathcal{O}\left(B^{2}\right) .
$$

All Landé factors are listed in the last column of Table A.1. 


\begin{tabular}{c|c|c}
${ }^{3}$ He level & $\hat{E} / h(\mathrm{GHz})$ & $\hat{g}_{F}$-factor \\
\hline $3^{3} \mathrm{~S}, \mathrm{~F}=1 / 2$ & 4.37289 & 2.66888 \\
$3^{3} \mathrm{~S}, \mathrm{~F}=3 / 2$ & -2.18645 & 1.3356 \\
\hline $2^{3} \mathrm{P}_{0}, \mathrm{~F}=1 / 2$ & 32.23217 & -0.17635 \\
$2^{3} \mathrm{P}_{\mathrm{x}}, \mathrm{F}=3 / 2$ & 4.80841 & 1.41962 \\
$2^{3} \mathrm{P}_{1}, \mathrm{~F}=1 / 2$ & 4.13924 & 2.1795 \\
$2^{3} \mathrm{P}_{\mathrm{y}}, \mathrm{F}=3 / 2$ & -0.37235 & 1.38262 \\
$2^{3} \mathrm{P}_{2}, \mathrm{~F}=5 / 2$ & -2.15305 & 1.20129 \\
\hline $2^{3} \mathrm{~S}, \mathrm{~F}=1 / 2$ & 4.49313 & 1.3356 \\
$2^{3} \mathrm{~S}, \mathrm{~F}=3 / 2$ & -2.24657 & 2.66888 \\
\hline \hline & & \\
& &
\end{tabular}

Table A.1: List of computed He level energy terms at $B=0:{ }^{*} E_{\mathrm{S}} / h, E_{\mathrm{P}} / h$, and $E_{\mathrm{S}} / h$ for ${ }^{3} \mathrm{He}$ (upper part), and $E_{\mathrm{P}}^{(4)} / h$ for ${ }^{4} \mathrm{He}$ (lower part). The third column lists the

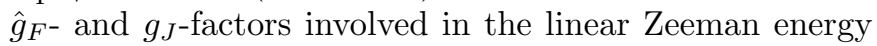
shifts for these levels.

\begin{tabular}{|c|c|c|c|c|c|}
\hline \multicolumn{2}{|c|}{$706.5 \mathrm{~nm}$ transition } & \multicolumn{2}{|c|}{$1083 \mathrm{~nm}$ transition } & \multicolumn{2}{|c|}{ levels } \\
\hline line & ${ }^{*} \epsilon_{n} / h, \mathrm{GHz}$ & line & $\epsilon_{n} / h, \mathrm{GHz}$ & $2^{3} \mathrm{P}$ & ${ }^{*} F, F$ \\
\hline${ }^{*} \mathrm{C}_{1}$ & 0 & $\mathrm{C}_{1}$ & 0 & $2^{3} \mathrm{P}_{y}$ & $1 / 2$ \\
\hline${ }^{*} \mathrm{C}_{2}$ & -4.51159 & $\mathrm{C}_{2}$ & 4.51159 & $2^{3} \mathrm{P}_{1}^{3}$ & $1 / 2$ \\
\hline${ }^{*} \mathrm{C}_{3}$ & -4.77864 & $\mathrm{C}_{3}$ & 4.959 & $2^{3} \mathrm{P}_{2}$ & $3 / 2$ \\
\hline${ }^{*} \mathrm{C}_{4}$ & -5.18076 & $\mathrm{C}_{4}$ & 5.18076 & $2^{3} \mathrm{P}_{x}$ & $1 / 2$ \\
\hline${ }^{*} \mathrm{C}_{5}$ & -6.55934 & $\mathrm{C}_{5}$ & 6.7397 & $2^{3} \mathrm{P}_{y}$ & $3 / 2$ \\
\hline${ }^{*} \mathrm{C}_{6}$ & -11.07093 & $\mathrm{C}_{6}$ & 11.25129 & $2^{3} \mathrm{P}_{1}$ & $3 / 2$ \\
\hline${ }^{*} \mathrm{C}_{7}$ & -11.7401 & $\mathrm{C}_{7}$ & 11.92046 & $2^{3} \mathrm{P}_{x}$ & $3 / 2$ \\
\hline${ }^{*} \mathrm{C}_{8}$ & -32.60452 & $\mathrm{C}_{8}$ & 32.60452 & $2^{3} \mathrm{P}_{0}$ & $1 / 2$ \\
\hline${ }^{*} \mathrm{C}_{9}$ & -39.16386 & $\mathrm{C}_{9}$ & 39.34422 & $2^{3} \mathrm{P}_{0}$ & $3 / 2$ \\
\hline line & ${ }^{*} \epsilon_{n}^{(4)} / h, \mathrm{GHz}$ & line & $\epsilon_{n}^{(4)} / h, \mathrm{GHz}$ & $2^{3} \mathrm{P}$ & $* J, J$ \\
\hline$* \mathrm{D}_{2}$ & -5.33963 & $\mathrm{D}_{2}$ & 38.53362 & $2^{3} \mathrm{P}_{2}$ & 1 \\
\hline${ }^{*} \mathrm{D}_{1}$ & -7.6308 & $\mathrm{D}_{1}$ & 40.82479 & $2^{3} \mathrm{P}_{1}$ & 1 \\
\hline${ }^{*} \mathrm{D}_{0}$ & -37.24775 & $\mathrm{D}_{0}$ & 70.44174 & $2^{3} \mathrm{P}_{0}$ & 1 \\
\hline
\end{tabular}

three considered states. Absolute optical transition energies cannot be inferred. However, energy or frequency differences between optical transitions can be computed from the level energies of Table A.1. The results are displayed in Table A.2 as a list of computed null-field frequency offsets (or frequencies, for compactness) ${ }^{*} \hat{\epsilon} / h$ (resp. $\hat{\epsilon} / h$ ) from the highest- (resp. lowest-) energy component of the $706.5 \mathrm{~nm}$ (resp. $1083 \mathrm{~nm}$ ) transition, for both isotopes.

For ${ }^{3} \mathrm{He}$ they are written as

$$
\begin{aligned}
{ }^{*} \epsilon_{j k} & =\left[{ }^{*} E_{\mathrm{S}}\left({ }^{*} \mathrm{~A}_{k}\right)-{ }^{*} E_{\mathrm{S}}\left({ }^{*} \mathrm{~A}_{5}\right)\right]-\left[E_{\mathrm{P}}\left(\mathrm{B}_{j}\right)-E_{\mathrm{P}}\left(\mathrm{B}_{7}\right)\right] \\
\epsilon_{i j} & =\left[E_{\mathrm{P}}\left(\mathrm{B}_{j}\right)-E_{\mathrm{P}}\left(\mathrm{B}_{7}\right)\right]-\left[E_{\mathrm{S}}\left(\mathrm{A}_{i}\right)-E_{\mathrm{S}}\left(\mathrm{A}_{5}\right)\right] .
\end{aligned}
$$

The transition frequencies of ${ }^{*} \mathrm{C}_{n}$ and $\mathrm{C}_{n}$ are exactly opposite when they involve the same $F=1 / 2$ hyperfine level as ${ }^{*} \mathrm{C}_{1}$ and $\mathrm{C}_{1}$, namely for $n=2,4$, and 8 (see the last column in Table A.2

For ${ }^{4} \mathrm{He}$ the line splittings solely result from the finestructure of the $2^{3} \mathrm{P}$ level. Their positions with respect to the $706.5 \mathrm{~nm}{ }^{3} \mathrm{He}$ lines are computed using the average of the two published values of isotopic shift differences $\Delta E$ between level energies of ${ }^{4} \mathrm{He}$ and ${ }^{3} \mathrm{He}$, namely:

$$
{ }^{*} \Delta=\Delta E\left(3^{3} \mathrm{~S}_{1}\right)-\Delta E\left(2^{3} \mathrm{P}_{0}\right)=594.106 \mathrm{MHz}
$$

according to [40, Table 2], and:

$$
{ }^{*} \Delta=594.658 \mathrm{MHz}
$$

according to 31, Table 1].

The computation of line positions listed in Table A.2 for the ${ }^{*} \mathrm{D}_{n}$ components involves this difference in energy terms

$$
\begin{aligned}
{ }^{*} \epsilon_{j k}^{(4)}= & {\left[{ }^{*} E_{\mathrm{S}}^{(4)}\left({ }^{*} \mathrm{Y}_{k}\right)-{ }^{*} E_{\mathrm{S}}\left({ }^{*} \mathrm{~A}_{5}\right)\right] } \\
& -\left[E_{\mathrm{P}}^{(4)}\left(\mathrm{Z}_{j}\right)-E_{\mathrm{P}}\left(\mathrm{B}_{7}\right)\right]-{ }^{*} \Delta
\end{aligned}
$$

where the $\mathrm{B}_{7}-{ }^{*} \mathrm{~A}_{5}$ transition $\left({ }^{*} \mathrm{C}_{1}\right.$ component) at $B=$ 0 is used as a reference for all frequency offsets. The values in Eqs. A.6 and A.7 differ by an amount which can safely be neglected in this work but may rather easily be experimentally settled.

Tables A.3 and A.5 compile the null-field values of the transition matrix elements for the $706.5 \mathrm{~nm}$ line for both isotopes.

Table A.4 lists some notations used in this article for the three involved levels of both isotopes.

The low-fields variations for the $3^{3} \mathrm{~S}$ and $2^{3} \mathrm{~S}$ states are simply given by the linear Zeeman terms

$$
{ }^{*} E_{\mathrm{S}}^{(4)}={ }^{*} g_{S}{ }^{*} m_{S} \mu_{B} B, E_{\mathrm{S}}^{(4)}(B)=g_{S} m_{S} \mu_{B} B,
$$

where ${ }^{*} g_{S} \approx g_{S}=2.00224$ [18, Table 5].

The effective Hamiltonian of Ref. [17] yields accurate energy differences only between sublevels of each of the 


\begin{tabular}{c|c|ccc} 
& & ${ }^{*} \mathrm{Y}_{1}$ & ${ }^{*} \mathrm{Y}_{2}$ & ${ }^{*} \mathrm{Y}_{3}$ \\
\hline & $m$ & -1 & 0 & 1 \\
\hline $\mathrm{Z}_{1}$ & -2 & $1 / 3$ & & \\
$\mathrm{Z}_{2}$ & -1 & $1 / 6$ & $1 / 6$ & \\
$\mathrm{Z}_{3}$ & 0 & $1 / 18$ & $2 / 9$ & $1 / 18$ \\
$\mathrm{Z}_{4}$ & 1 & & $1 / 6$ & $1 / 6$ \\
$\mathrm{Z}_{5}$ & 2 & & & $1 / 3$ \\
\hline $\mathrm{Z}_{6}$ & -1 & $1 / 6$ & $1 / 6$ & \\
$\mathrm{Z}_{7}$ & 0 & $1 / 6$ & 0 & $1 / 6$ \\
$\mathrm{Z}_{8}$ & 1 & & $1 / 6$ & $1 / 6$ \\
\hline $\mathrm{Z}_{9}$ & 0 & $1 / 9$ & $1 / 9$ & $1 / 9$
\end{tabular}

Table A.3: List of ${ }^{4} \mathrm{He}$ transition matrix elements ${ }^{*} T_{j k}^{(4)}$ between sublevels $\mathrm{Z}_{j}$ and ${ }^{*} \mathrm{Y}_{k}$ for $B=0$. The color (online) indicates the light polarisation vector: red for $\sigma_{+}$, black for $\pi$, and blue for $\sigma_{-}$. It corresponds to the angular momentum change between the $m$-values $m_{J}$ and ${ }^{*} m_{J}$. The empty cells in the table correspond to forbidden dipolar electric transitions $(|\Delta m|>1)$ and therefore null values of ${ }^{*} T_{j k}^{(4)}$.

\begin{tabular}{ccc|ccc} 
atom & state & sublevel & pop. & $\begin{array}{c}\text { density } \\
\mathrm{m}^{-3}\end{array}$ & $\begin{array}{c}\text { vel. distr. } \\
(\mathrm{m} / \mathrm{s})^{-1}\end{array}$ \\
\hline${ }^{3} \mathrm{He}$ & $2^{3} \mathrm{~S}$ & $\mathrm{~A}_{i}$ & $a_{i}$ & $n_{\mathrm{m}} a_{i}$ & $\phi_{i}\left(v_{z}\right)$ \\
${ }^{3} \mathrm{He}$ & $2^{3} \mathrm{P}$ & $\mathrm{B}_{j}$ & $b_{j}$ & $n_{\mathrm{m}} b_{j}$ & $\phi_{j}^{\prime}\left(v_{z}\right)$ \\
${ }^{3} \mathrm{He}$ & $3^{3} \mathrm{~S}$ & ${ }^{*} \mathrm{~A}_{k}$ & ${ }^{*} a_{k}$ & $n_{\mathrm{m}}{ }^{*} a_{k}$ & \\
\hline${ }^{4} \mathrm{He}$ & $2^{3} \mathrm{~S}$ & $\mathrm{Y}_{i}$ & $y_{i}$ & $n_{\mathrm{m}}^{(4)} y_{i}$ & $\phi_{i}^{(4)}\left(v_{z}\right)$ \\
${ }^{4} \mathrm{He}$ & $2^{3} \mathrm{P}$ & $\mathrm{Z}_{j}$ & $z_{j}$ & $n_{\mathrm{m}}^{(4)} z_{j}$ & $\phi_{j}^{(4)}\left(v_{z}\right)$ \\
${ }^{4} \mathrm{He}$ & $3^{3} \mathrm{~S}$ & ${ }^{*} \mathrm{Y}_{k}$ & ${ }^{*} y_{k}$ & $n_{\mathrm{m}}^{(4)} y_{k}$ &
\end{tabular}

\begin{tabular}{c|cccc|cc} 
& ${ }^{*} \mathrm{~A}_{1}$ & ${ }^{*} \mathrm{~A}_{2}$ & ${ }^{*} \mathrm{~A}_{3}$ & ${ }^{*} \mathrm{~A}_{4}$ & ${ }^{*} \mathrm{~A}_{5}$ & ${ }^{*} \mathrm{~A}_{6}$ \\
\hline 1 & $1 / 3$ & & & & & \\
2 & $2 / 15$ & $1 / 5$ & & & & \\
3 & $1 / 30$ & $1 / 5$ & $1 / 10$ & & & \\
4 & & $1 / 10$ & $1 / 5$ & $1 / 30$ & & \\
5 & & & $1 / 5$ & $2 / 15$ & & \\
6 & & & & $1 / 3$ & & \\
\hline 7 & .19465 & .12977 & & & .00891 & \\
8 & .12977 & .02163 & .17302 & & .00594 & .00297 \\
9 & & .17302 & .02163 & .12977 & .00297 & .00594 \\
10 & & & .12977 & .19465 & & .00891 \\
\hline 11 & .07296 & .04864 & .02432 & & .06247 & .12494 \\
12 & & .02432 & .04864 & .07296 & .12494 & .06247 \\
\hline 13 & .00535 & .00357 & & & .32442 & \\
14 & .00357 & .00059 & .00475 & & .21628 & .10814 \\
15 & & .00475 & .00059 & .00357 & .10814 & .21628 \\
16 & & & .00357 & .00535 & & .32442 \\
\hline 17 & & .031232 & .06247 & .09370 & .09728 & .04864 \\
18 & .09370 & .06247 & .03123 & & .04864 & .09728
\end{tabular}

Table A.4: List of notations used to characterise local quantities for both He isotopes in the relevant states and sublevels (first three columns). The (true) populations in the $2^{3} \mathrm{~S}$ state verify $\sum a_{i}=\sum y_{i}=1$, while the (pseudo) populations in the excited states (column 4) are the coefficients linking number densities in a sublevel (column 5) to the total number density $n_{\mathrm{m}}$ or $n_{\mathrm{m}}^{(4)}$ in the $2^{3} \mathrm{~S}$ state. Column 6 lists the notations for the normalised velocity distributions, where $v_{z}$ is the atomic velocity component on the pump (and probe) light propagation axis.

Table A.5: List of ${ }^{3} \mathrm{He}$ transition matrix elements ${ }^{*} T_{j k}$ between sublevels $\mathrm{B}_{j}$ and ${ }^{*} \mathrm{~A}_{k}$ for $B=0$. The first column contains the index $j$ of the lower sublevel $\mathrm{B}_{j}$. The color (online) indicates the light polarisation vector: red for $\sigma_{+}$, black for $\pi$, and blue for $\sigma_{-}$. It corresponds to the angular momentum change between the half-integer $\hat{m}$-values, $m_{F}$ and ${ }^{*} m_{F}$, not displayed in this table. As in Table A.3 the empty cells correspond to null values of ${ }^{*} T_{j k}$ (forbidden dipolar electric transitions, $|\Delta F|>1$ or $\left.\left|\Delta m_{F}\right|>1\right)$. 


\section{B Doppler widths}

The generic Doppler width parameters are defined as

$$
\hat{\Delta}=\hat{\nu} \sqrt{2 k_{\mathrm{B}} T / \hat{M} c^{2}}
$$

where $\hat{\nu}$ is the optical transition frequency, $k_{\mathrm{B}}$ the Boltzmann constant, $T$ the gas temperature, and $\hat{M}$ the atomic mass. Small relative variations of $\hat{\nu}$ between line components or isotopes are usually neglected, and values of $\hat{\Delta}$ at room temperature are listed in Table B.1. The Doppler FWHM values are $2 \hat{\Delta} \sqrt{\ln 2}$.

\begin{tabular}{c|cc} 
& ${ }^{3} \mathrm{He}$ & ${ }^{4} \mathrm{He}$ \\
\hline $1083 \mathrm{~nm}$ & $\Delta_{3}=1.1875$ & $\Delta_{4}=1.0284$ \\
$706.5 \mathrm{~nm}$ & ${ }^{*} \Delta_{3}=1.8203$ & ${ }^{*} \Delta_{4}=1.5764$
\end{tabular}

Table B.1: Values of the Doppler width parameters, generically noted $\hat{\Delta}$, in $\mathrm{GHz}$, for ${ }^{3} \mathrm{He}$ and ${ }^{4} \mathrm{He}$ and for both optical transitions. They are computed for a temperature $T=300 \mathrm{~K}$.

\section{Magnetic-field coils}

The system of coils was designed to provide a field with sufficient uniformity over the volume of a cylindrical experimental cell with length up to $30 \mathrm{~cm}$ and diameter up to $6 \mathrm{~cm}$. A combination of geometrical constraints chosen for ease of construction and numerical optimisation of parameters have resulted in the actual system.

The axial $B_{z}$ field was generated by a set of six square coils (external side length: $42 \mathrm{~cm}$ ) wound on aluminium alloy frames made from welded U-profile $(20 \times 20 \times 20 \times 2$ $-\mathrm{mm}$ ) assembled by brass threaded rods near the corners (Fig. C.1). Their optimised distances from centre $( \pm 5.9 \mathrm{~cm}, \pm 19.1 \mathrm{~cm}$ and $\pm 37.8 \mathrm{~cm})$ and numbers of turns (85, 100, and 224, respectively) provide a fairly uniform field with a limited overall size and easy access to all parts of the system.

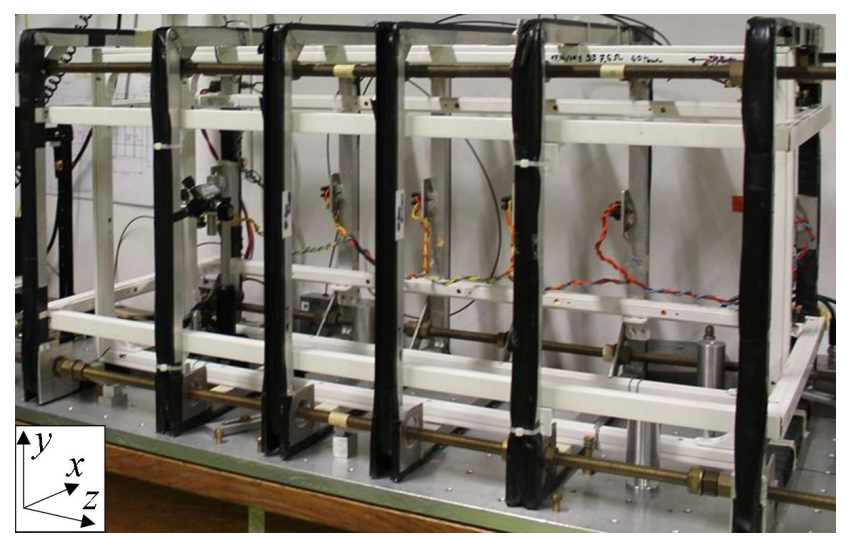

Figure C.1: View of the assembled set of magnetic field coils (see text).
The computed relative variations of the main field in several planes are displayed in Fig. C.2. With 0.8-mmdiameter wire, the total resistance was $27 \Omega$ and fields up to $4 \mathrm{mT}\left(B_{z}=0.85 \mathrm{mT} / \mathrm{A}\right)$ could be achieved without forced cooling.
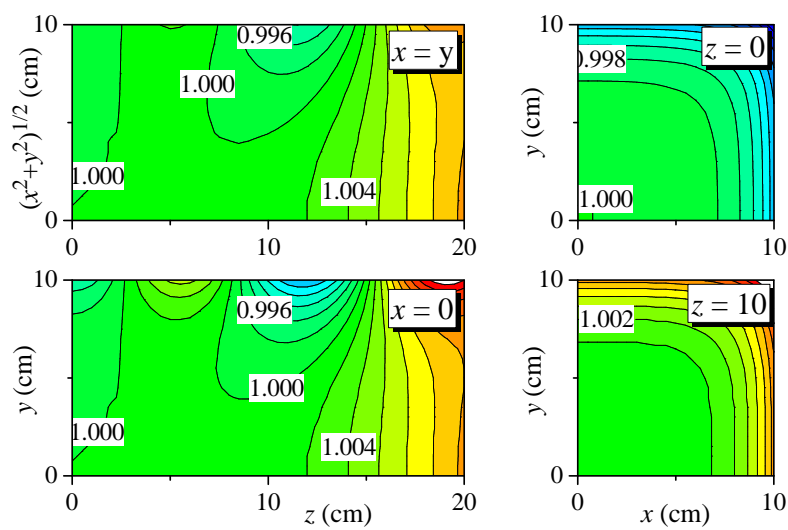

Figure C.2: Computed field maps for the set of six square coils. $B_{z}(\mathbf{r}) / B_{z}(0)$ maps are displayed in one quadrant of diagonal and vertical planes including the $z$-axis (with contours every $0.2 \%$ ) and of vertical planes at the coil centre and $10 \mathrm{~cm}$ away (with contours every $0.1 \%$ ).

The transverse field components were generated by pairs of rectangular coils comprising 40 turns each. They were wound on PVC wireways $\left(20 \times 20 \mathrm{~mm}^{2}\right)$ assembled using plastic corner angle brackets. Their outside width just fit inside the $36-\mathrm{cm}$ clearance of the $B_{z}$ coils to which they were attached. Their outside lengths were similar to the length of the $B_{z}$ coil set, with the $B_{x}$ coils fitting inside the $B_{y}$ coils (Fig. C.1). Selected computed field maps are shown in Figs. C.3 and C.4. With this config-
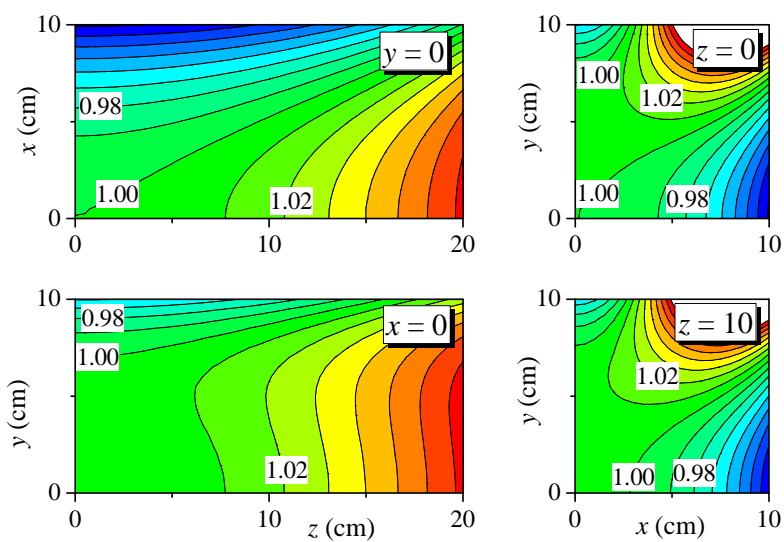

Figure C.3: Computed field maps for $B_{x}$ coil pair, with outside length $73.5 \mathrm{~cm}$ and mean separation $17 \mathrm{~cm}$. $B_{x}(\mathbf{r}) / B_{x}(0)$ maps are displayed in one quadrant of horizontal and vertical planes including the $z$-axis and of vertical planes at the coil centre and $10 \mathrm{~cm}$ away (contours every $1 \%$ in all plots).

uration, the generated fields were $B_{y}=0.146 \mathrm{mT} / \mathrm{A}$ and 

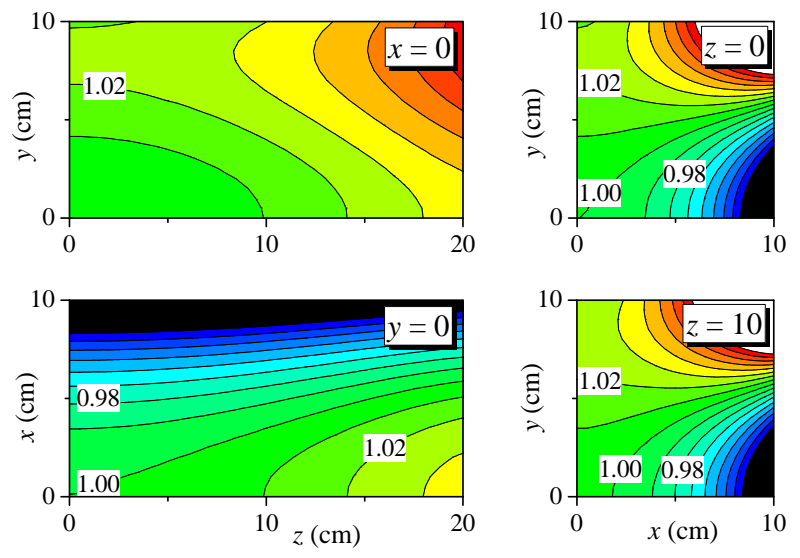

Figure C.4: Computed field maps for $B_{y}$ coil pair, with outside length $78.5 \mathrm{~cm}$ and mean separation $24 \mathrm{~cm}$. $B_{y}(\mathbf{r}) / B_{y}(0)$ maps are displayed in one quadrant of horizontal and vertical planes including the $z$-axis and of vertical planes at the coil centre and $10 \mathrm{~cm}$ away (contours every $1 \%$ in all plots).

$B_{x}=0.171 \mathrm{mT} / \mathrm{A}$. With 0.4-mm-diameter wire, the total resistance was about $16 \Omega$ and transverse fields up to $2 \mathrm{mT}$ could be achieved. The difference of their field maps and strengths mainly resulted from the difference in coil separations, chosen as trade-offs between convenience and field homogeneity. The latter was poorer than that of $B_{z}$ but was sufficient for Earth's field compensation of transverse components or for applying a moderate transverse field on the 5- to 10-cm-long experimental cells.

\section{Pump wavelength measure- ments}

Simultaneous recordings of the $1083 \mathrm{~nm}$ pump wavelength and of saturated absorption signals from the $\mathrm{C}_{\text {lock }}$ cell (Fig. 4) are reported here. The probe laser and experimental cell were not used, and the photodetector $\mathrm{P}_{2}$ was replaced with the collimator of the wavemeter input fibre.

Figure D.1 displays a long-term recording of the wavemeter readout $\lambda$ with the pump locked on the $D_{2}$ transition, of wavelength in vacuum $\lambda_{2}=1083.33064 \mathrm{~nm}$ (from [30, see footnote to Eq. 32). The difference $\lambda-\lambda_{2}$ was of order 1.6 to $2.6 \mathrm{pm}$, i.e. 410 to $670 \mathrm{MHz}$ in this example. Short-term drifts (see the blow-up) during typical scan times did not exceed 10 to $20 \mathrm{MHz}$, with a sub-MHz precision for smoothed data. The sudden noise increase and readout jump near the end of the recording were induced by small movements of the wavemeter input fibre.

Figure $\mathrm{D} .2$ displays saturated absorption data and fitting steps for a frequency scan over the $\mathrm{D}_{1}$ to $\mathrm{C}_{8}$ lines in a mixture cell. For convenience data processing consisted of two steps: the demodulated signals (left) were fit using Gaussian profiles and the residues (right) were fit using Lorentzian profiles. The positions of the saturated absorption dips, which had typical widths of $8 \mathrm{pm}$, i.e.

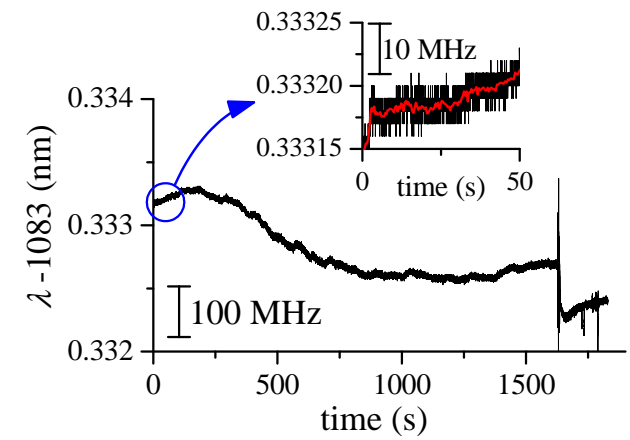

Figure D.1: Recording of wavemeter data for the 1083nm pump DL locked on the ${ }^{4} \mathrm{He} \mathrm{D}_{2}$ line $\left(\lambda_{2}\right.$ : see text). The inset is a blow-up $(10 \times)$ of part of the recording, with raw data (black, $10 \mathrm{~ms}$ sampling) and smoothed data (red, 20-point running averages).

$20 \mathrm{MHz}$, were thus precisely inferred.
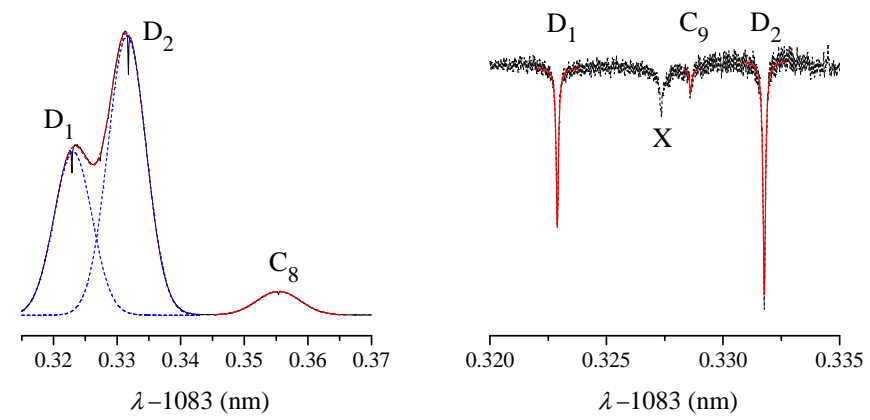

Figure D.2: Left: Demodulated saturated absorption signal from $\mathrm{P}_{1}$ (see Fig. 4 with a modulated discharge amplitude in $\mathrm{C}_{\text {lock }}(0.27+0.27$ mbar mixture). Sums of Gaussian profiles (blue and red dotted lines) were used to model thermal velocity distributions. Right: Expanded difference of the signals and the Gaussian fits for the left half of the spectrum. Three Lorentzian profiles were used to fit the saturated absorption dips. Atomic lines are labelled, $\mathrm{X}$ is the crossover resonance of $\mathrm{D}_{1}$ and $\mathrm{D}_{2}$.

Series of recordings comprising three back and forth scans were repeated on four different days using two gas mixture cells. The line position fits from each scan were combined to compute the three line splittings $\mathrm{D}_{1}-\mathrm{D}_{2}, \mathrm{D}_{2}-$ $\mathrm{C}_{8}$, and $\mathrm{C}_{9}-\mathrm{C}_{8}$ plotted in Figs. D.3 to c (the wavelengths were converted to frequencies and to frequency differences in a straightforward way). As was observed for the probe laser scans (Sect. 4.5), a significant scatter of results was found for these experiments. Figs. D.3 to f display the results of the same recordings, where the wavemeter readout values were replaced with the laser frequency control voltage data in the horizontal axes of Fig. D.2. The scaling factor between frequency and voltage differences was evaluated as the average of the ratios of the theoretical frequency splittings (the dashed lines in Figs. D.3 to c) to the measured voltage splittings (in Figs. D.3 to f).

The scatter of the voltage splittings was significantly 

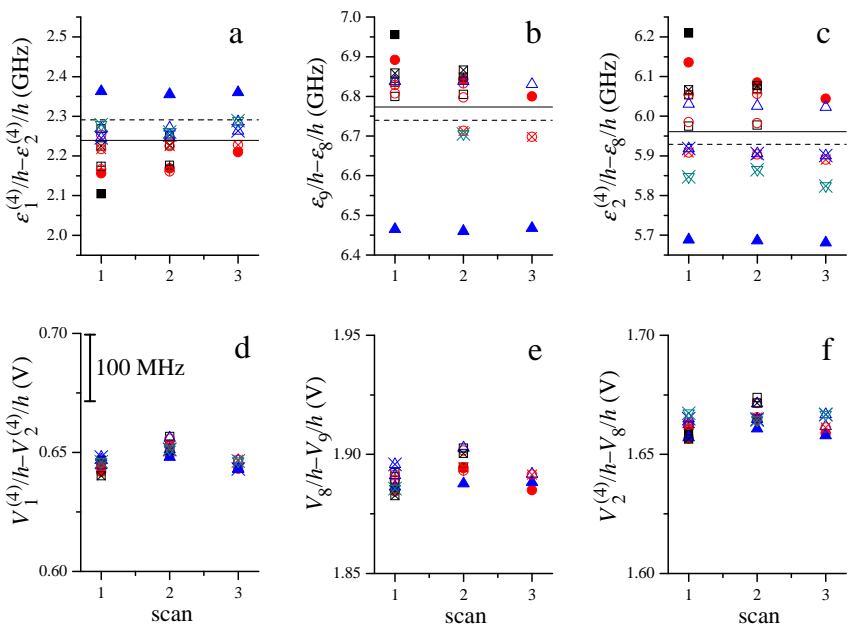

Figure D.3: Results of saturated absorption data yielding fit positions of the $\mathrm{C}_{8}, \mathrm{D}_{2}, \mathrm{C}_{9}$, and $\mathrm{D}_{1}$ lines for series of 3 repeated scans (see Fig. D.2). Top (a to c): frequency splittings. Bottom ( $\mathrm{d}$ to $\mathrm{f}$ ): frequency control voltage differences. a,d: for the $\mathrm{D}_{1}-\mathrm{D}_{2}$ interval. b,e: for the $\mathrm{C}_{8}$ $\mathrm{C}_{9}$ interval. $\mathrm{c}$,f: for the $\mathrm{C}_{8}-\mathrm{D}_{2}$ interval. The solid and dashed lines in graphs a to $\mathrm{c}$ are the averaged data and computed values, respectively.

lower than that inferred from wavelength data, as appears on the statistical summary of the plotted results listed in Table D.1 A 4 to 8-fold reduction of the standard deviation of the three probed line splittings is evidenced, with a significant reduction of the differences from expected values (lines 4 to 6 vs. lines 1 to 3 in Table D.1.

\begin{tabular}{c|c|c|c|c|c}
$\begin{array}{c}\text { line } \\
\text { names }\end{array}$ & $\begin{array}{c}\text { data } \\
\text { type }\end{array}$ & $\begin{array}{c}\text { av. }- \text { th. } \\
(\mathrm{MHz})\end{array}$ & $\begin{array}{c}\mathrm{SD} \\
(\mathrm{MHz})\end{array}$ & $\begin{array}{c}\mathrm{SE} \\
(\mathrm{MHz})\end{array}$ & $N_{\mathrm{s}}$ \\
\hline$C_{9}-C_{8}$ & $\lambda$ & 33.7 & 135 & 28 & 23 \\
$D_{2}-C_{8}$ & $\lambda$ & 31.9 & 131 & 24 & 29 \\
$D_{2}-D_{1}$ & $\lambda$ & -51.5 & 61 & 11 & 23 \\
\hline$C_{9}-C_{8}$ & $V$ & -15.9 & 20.7 & 4.2 & 23 \\
$D_{2}-C_{8}$ & $V$ & -15.8 & 16.1 & 3 & 29 \\
$D_{2}-D_{1}$ & $V$ & 11.7 & 15.5 & 2.9 & 23 \\
\hline
\end{tabular}

Table D.1: Statistics for frequency splitting data compiled in Fig. D.3. Columns 3 to 5 contain the differences between the averages of the fit frequencies and the computed values, the standard deviation and the standard error of the fit frequencies. The first three lines are data from wavemeter readings, the last 3 lines from control voltage values (see text, data types $\lambda$ or $V$ ). $N_{\mathrm{s}}$ is the number of scans used in the averages.

The results of these series of recordings are thus affected by the measurement errors of the wavemeter in a way similar to those of Sect. 4.5. Moreover, since the pump diode laser frequency is known to be reliably controlled by its temperature (therefore, by the control voltage in these experiments), the large reduction of errors in line splittings expressed as functions of this voltage further demonstrates that the frequency dependence of the wavemeter errors was the limiting factor for the precision of our Dopplerfree measurement on the $706.5 \mathrm{~nm}$ transition.

\section{References}

[1] T. Gentile, P.-J. Nacher, B. Saam, and T. Walker. 'Optically polarized ${ }^{3}$ He.' Rev. Mod. Phys., 89:045004 (2017).

[2] A. Dantan, G. Reinaudi, A. Sinatra, F. Laloë, et al. 'Long-lived quantum memory with nuclear atomic spins.' Phys. Rev. Lett., 95(12):123002 (2005).

[3] Y. Zhan, X. Peng, S. Li, L. Zhang, et al. 'Observation of transferred dressed spin effect via metastabilityexchange collisions in He-3 atoms.' Appl. Phys. B, 125(9):170 (2019).

[4] S. Li, Y. Zhan, X. Peng, J. Chen, et al. 'Linear and nonlinear coherence transfer of resonant nuclear-spindressed effect via metastability-exchange collisions.' J. Phys. B: At. Mol. Opt. Phys., 53(6):065202 (2020),

[5] H. Gilles, J. Hamel, and B. Cheron. 'Laser pumped He-4 magnetometer.' Rev. Sci. Instrum., 72(5):22532260 (2001).

[6] F. Beato, E. Belorizky, E. Labyt, M. Le Prado, et al. 'Theory of a ${ }^{4} \mathrm{He}$ parametric-resonance magnetometer based on atomic alignment.' Phys. Rev. A, 98:053431 (2018).

[7] G. Lieb, T. Jager, A. Palacios-Laloy, and H. Gilles. 'All-optical isotropic scalar ${ }^{4} \mathrm{He}$ magnetometer based on atomic alignment.' Rev. Sci. Instrum., 90(7):075104 (2019).

[8] M. Kodaira and T. Watanabe. 'Collisional Transfer of Triplet Excitations between Helium Atoms.' J. Phys. Soc. Jpn., 27(5):1301-1309 (1969).

[9] D. Vrinceanu, S. Kotochigova, and H. Sadeghpour. 'Pressure broadening and shift of $\mathrm{He}\left(2^{3} \mathrm{P}_{0,1,2}\right)$ He $\left(2^{3} \mathrm{~S}\right)$ lines.' Phys. Rev. A, 69(2):022714 (2004),

[10] L. Schearer. 'Collision-Induced Mixing in the $2^{3} \mathrm{P}$ Levels of Helium.' Phys. Rev., 160(1):76-80 (1967).

[11] A. Dia, P.-J. Nacher, M. Abboud, and G. Tastevin. 'Spectroscopic study of collisions in the $2^{3} \mathrm{P}$ state of ${ }^{3} \mathrm{He}$ and ${ }^{4} \mathrm{He}$ in low pressure gas discharges.' In ' 25 ème congrès général de la Société Française de Physique,' Nantes, France (2019).

[12] M. Fred, F. Tomkins, J. Brody, and M. Hamermesh. 'The Spectrum of ${ }^{3}$ HeI.' Phys. Rev., 82(3):406-421 (1951).

[13] J. Brochard, H. Chantrel, and P. Jacquinot. 'Étude des niveaux de ${ }^{3} \mathrm{He}$ par spectroscopie optique à très basse température.' J. Phys. Radium, 19(5):515-522 (1958).

[14] J. Su and J. Nicol. 'Measurements of self broadening of the triplet line $\lambda 706.5 \mathrm{~nm}$ in helium.' J. Phys. B: At. Mol. Opt. Phys., 23(14):2215-2222 (1990).

[15] J. Su and J. Nicol. 'Pressure shift of the helium triplet line $\lambda 706.5$ nm.' J. Phys. B: At. Mol. Opt. Phys., 26(2):255-260 (1993). 
[16] M. Andersson and L. Pendrill. 'Improved Measurements of the Hyperfine Structure of the $1 \mathrm{~s} 3 \mathrm{~s}^{3} \mathrm{~S}_{1}$ State of He-3.' Phys. Scripta, 30(6):403-406 (1984).

[17] E. Courtade, F. Marion, P.-J. Nacher, G. Tastevin, et al. 'Magnetic field effects on the $1083 \mathrm{~nm}$ atomic line of helium.' Eur. Phys. J. D, 21(1):25-55 (2002)

[18] Q. Wu. 'Precision calculation of electronic $g_{J}$ factors for $n^{3} \mathrm{~S}_{1}$ states of ${ }^{4} \mathrm{He}$ and ${ }^{7} \mathrm{Li}^{+}$ion.' J. Phys. B: At. Mol. Opt. Phys., 39(20):4213-4219 (2006).

[19] R. Hilborn. 'Einstein coefficients, cross-sections, $f$ values, dipole-moments, and all that.' Am. J. Phys., 50(11):982-986 (1982).

[20] M. Millard, P. Yaney, B. Ganguly, and C. DeJoseph. 'Diode laser absorption measurements of metastable helium in glow discharges.' Plasma Sources Sci. Technol., 7(3):389-394 (1998).

[21] R. Hilborn. 'Einstein coefficients, cross sections, $f$ values, dipole moments, and all that.' ArXiv:physics/0202029.

[22] A. Nikiel-Osuchowska, G. Collier, B. Glowacz, T. Palasz, et al. 'Metastability exchange optical pumping of ${ }^{3} \mathrm{He}$ gas up to hundreds of millibars at 4.7 Tesla.' Eur. Phys. J. D, 67(9):200 (2013).

[23] M. Pinard, C. Aminoff, and F. Laloë. 'Velocityselective optical pumping and Doppler-free spectroscopy.' Phys. Rev. A, 19(6):2366-2370 (1979)

[24] W. Wiese and J. Fuhr. 'Accurate Atomic Transition Probabilities for Hydrogen, Helium, and Lithium.' J. Phys. Chem. Ref. Data, 38:565-719 (2009)

[25] W. Demtröder. Laser Spectroscopy, volume 2, chapter 2, pages 83-147. Springer (2015). ISBN 978-3662-44640-9. doi:10.1007/978-3-662-44641-6.

[26] P.-J. Nacher and M. Leduc. 'Optical pumping in ${ }^{3}$ He with a laser.' J. Phys-Paris, 46(12):2057-2073 (1985)

[27] M. Batz, P.-J. Nacher, and G. Tastevin. 'Fundamentals of metastability exchange optical pumping in helium.' J. Phys. Conf. Ser., 294(1):012002 (2011).

[28] N. Bigelow, P.-J. Nacher, and M. Leduc. 'Accurate optical measurement of nuclear polarization in optically pumped ${ }^{3} \mathrm{He}$ gas.' J. Phys. II, 2(12):2159-2179 (1992).

[29] C. Talbot, M. Batz, P.-J. Nacher, and G. Tastevin. 'An accurate optical technique for measuring the nuclear polarisation of ${ }^{3}$ He gas.' J. Phys. Conf. Ser., 294:012008 (2011).

[30] A. Kramida, Yu. Ralchenko, J. Reader, and NIST ASD Team. NIST Atomic Spectra Database (ver. 5.8), [Online]. Available: https://physics.nist.gov/asd [2020, December 7]. National Institute of Standards and Technology, Gaithersburg, MD. (2020).

[31] D. Morton, Q. Wu, and G. Drake. 'Energy levels for the stable isotopes of atomic helium $\left({ }^{4} \mathrm{He} \mathrm{I}\right.$ and ${ }^{3} \mathrm{He}$ I).' Can. J. Phys., 84(2):83-105 (2006).

[32] K. Saleh, J. Millo, A. Didier, Y. Kersalé, et al. 'Frequency stability of a wavelength meter and applications to laser frequency stabilization.' Appl. Opt., 54(32):9446-9449 (2015).

[33] L. Couturier, I. Nosske, F. Hu, C. Tan, et al. 'Laser frequency stabilization using a commercial wavelength meter.' Rev. Sci. Instrum., 89(4):043103 (2018).

[34] K. Koenig, P. Imgram, J. Kraemer, B. Maass, et al. 'On the performance of wavelength meters: Part 2frequency-comb based characterization for more accurate absolute wavelength determinations.' Appl. Phys. B, 126(5):86 (2020).

[35] R. Whitley and C. Stroud. 'Double optical resonance.' Phys. Rev. A, 14(4):1498-1513 (1976).

[36] C. Cohen-Tannoudji, J. Dupont-Roc, and G. Grynberg. Atom-Photon Interactions: Basic Processes and Applications. Wiley, New York (1992). ISBN 978-0-471-29336-1.

[37] J. Mompart and R. Corbalan. 'Generalized Einstein B coefficients for coherently driven three-level systems.' Phys. Rev. A, 63(6):063810 (2001)

[38] B. Aurand, S. Kuschel, C. Rödel, M. Heyer, et al. 'Creating circularly polarized light with a phaseshifting mirror.' Opt. Express, 19(18):17151-17157 (2011).

[39] D. Bloch, G. Trenec, and M. Leduc. 'Isotope shift of the $2^{3} \mathrm{~S}_{1}-2^{3} \mathrm{P}$ transition in helium.' J. Phys. B: At. Mol. Opt. Phys., 18(6):1093-1100 (1985)

[40] G. Drake, W. Nörtershäuser, and Z. C. Yan. 'Isotope shifts and nuclear radius measurements for helium and lithium.' Can. J. Phys., 83(4):311-325 (2005). 